\title{
氨基酸功能化晶态多孔聚合物的研究进展
}

\author{
梅佩张媛媛* 冯霄* \\ (北京理工大学 化学与化工学院 前沿交叉科学研究院 北京 100081)
}

\begin{abstract}
摘要 以金属有机框架(metal-organic frameworks, MOFs)和共价有机框架(covalent organic frameworks, COFs)为代表的 晶态多孔聚合物, 具有高比表面积、多样的结构、开放的孔道、丰富的官能团、易功能化等特点, 在气体储存和分离、 催化、储能、光电器件等领域都有着广泛的应用前景. 氨基酸是构成多肽和蛋白质的基本结构单元, 不仅具有重要的 生物学功能, 还在药物生产、生物降解塑料、手性催化剂等工业应用中发挥重要作用. 将氨基酸引入到 MOFs 和 COFs 体系中, 可赋予其柔性化骨架、特殊的孔道环境、手性识别位点等特征, 并且在一定程度上提高框架材料的生物相容 性、可降解性, 进一步丰富晶态多孔聚合物的功能和应用. 本综述概括了利用氨基酸功能化 MOF 和 COF 材料的制备 策略, 主要包括以氨基酸及其衍生物作为构筑单元、骨架共价修饰氨基酸、以氨基酸作为调节剂; 并重点介绍了这些 材料在手性拆分、催化、吸附以及质子传导等领域的应用. 最后, 本综述分析了当前氨基酸功能化的晶态多孔聚合物 面临的困难与挑战, 并对其未来研究方向进行了展望.
\end{abstract}

关键词 晶态多孔聚合物; 金属有机框架; 共价有机框架; 氨基酸; 功能化

\section{Amino Acid Functionalized Crystalline Porous Polymers}

\author{
Mei, Pei Zhang, Yuanyuan* Feng, Xiao* \\ (School of Chemistry and Chemical Engineering, Advanced Research Institute of Multidisciplinary Science, \\ Beijing Institute of Technology, Beijing 100081, China)
}

\begin{abstract}
Metal-organic frameworks (MOFs) and covalent organic frameworks (COFs) are representative crystalline porous polymers. Due to their high surface area, high porosity, open channels, abundant functional groups and easy functionalization, they show great applications in gas storage and separation, catalysis, energy storage, photovoltaic devices, etc. Amino acids are the basic structural units that constitute peptides and proteins, which not only have important biological functions, but also play an important role in industrial applications such as pharmaceutical production, biodegradable plastics, and chiral catalysts. The introduction of amino acids into MOFs and COFs could endow them with diverse and flexible frameworks, special pore environment, and chiral sites, improving their biocompatibility and degradability to some extent and enriching their functions and applications. This review focuses on the progress of the amino acid functionalized MOFs and COFs, including their synthetic strategies, such as employing amino acids and their derivatives as building unit, covalent modification of amino acids onto the framework, and utilizing amino acids as modulators. The advantages and disadvantages of these strategies are compared and their challenges are discussed. In addition, we also introduce their applications in chiral separation, catalysis, adsorption and proton conduction. Finally, we summarize the current challenges in the preparation of amino acid functionalized crystalline porous polymers and outlook the future research direction in this field.
\end{abstract}

Keywords crystalline porous polymer; metal-organic frameworks; covalent organic frameworks; amino acid; functionalization

\section{1 引言}

晶态多孔聚合物是一类具有丰富结构和官能团、高 结晶度、高比表面积、发达孔道结构的功能多孔材料, 展 现出良好的应用前景, 近年来该领域取得了长足的发 展 $^{[1]}$. 其中, 金属有机框架(metal-organic frameworks, $\mathrm{MOFs}$ )或称多孔配位聚合物(porous coordination polymers, PCPs), 是由金属离子/团簇和有机配体组装形成 的无机有机杂化多孔材料 ${ }^{[2]}$; 共价有机框架(covalent organic frameworks, COFs)是由有机构筑单元通过共价 键组装而成的晶态多孔材料 ${ }^{[3]}$. 与传统的多孔材料相比, 晶态多孔聚合物具有以下特点: (1)丰富可设计的组成和 结构, 在网状化学的设计理念下可通过调控构筑单元及 其连接方式构筑具有不同结构特征的框架结构; (2)规整 有序的孔道结构, 且可对其孔径、形状、化学环境进行 调控; (3)易于官能化, 可通过官能化的构筑单元或合成 后修饰的策略实现骨架的功能化 ${ }^{[1 a]}$. 因此, 此类材料在

\footnotetext{
*E-mail: 6120190112@bit.edu.cn; fengxiao86@bit.edu.cn

Received June 22, 2020; published July 21, 2020.

Project supported by the National Natural Science Foundation of China (Nos. 21922502, 21674012).

项目受国家自然科学基金(Nos. 21922502, 21674012)资助.
} 
气体存储与分离 ${ }^{[4]}$ 、催化 ${ }^{[5]}$ 、传感 ${ }^{[6]}$ 、生物医药 ${ }^{[7]}$ 等领域 都有巨大的应用潜力. 近年来, 开发、设计生物分子功 能化的晶态多孔聚合物受到广泛关注. 将具有生物活性 的分子引入 $\mathrm{MOF}$ 和 $\mathrm{COF}$ 材料体系, 可进一步丰富其组 成和结构, 拓展其应用领域 ${ }^{[8]}$.

氨基酸是构成蛋白质和其它生物分子的基本单元, 在有机体中具有重要的作用 ${ }^{[9]}$. 目前已被发现存在于自 然界中的氨基酸约有 1000 种. 科学技术的进步不仅阐 明了氨基酸分子的生物学功能, 而且拓展了其在化工和 医药等领域的应用 ${ }^{[10]}$. 例如, 手性氨基酸可用于手性合 成, 以及作为手性催化剂的前驱体; 许多氨基酸具有医 用价值, 如精氨酸可用于肝昏迷急救, 谷氨酸可用于功 能性消化不良和心肌梗死等疾病的治疗, 有些氨基酸还 被用于癌症的诊断指标 ${ }^{[10]}$. 氨基酸以一定序列脱水缩 合可以组成多肽, 它们具有多种生物活性, 比如抗氧化 性、催化活性、抗菌性、抑制肿瘤、降低血脂等. 此外, 氨基酸结构除氨基和羧基外还包括丰富的侧链基团, 比 如甲基、氨基、羧基、羰基、吲哚基、咪唑基、苯基、 颈基等 ${ }^{[11]}$; 这些结构特点使得氨基酸与金属离子配位 或与其他官能团共价成键的方式更为多样化. 基于其丰 富的结构和功能, 氨基酸成为晶态多孔聚合物材料功能 化的理想选择.

在晶态多孔聚合物骨架中引入氨基酸单元, 可赋予 其特殊的性质和功能. 首先, 氨基酸多样的结构和反应 位点使得其作为分子单元构筑框架材料可以得到多样 的结构, 同时还可实现骨架的柔性化; 其次, 氨基酸可 以参与框架孔道尺寸和环境的调节, 赋予晶态多孔聚合 物特定的结构特征和物化性质; 此外, 通过氨基酸功能 化可在骨架中引入手性环境, 并用于分子识别、分离、 催化等; 最后, 氨基酸的引入可在一定程度上实现框架 材料的生物相容性、低毒性、可降解性. 近年来已有生 物型 MOFs 综述中涵盖部分相关内容 ${ }^{[8]}$, 但是专门针对 氨基酸功能化晶态多孔框架材料的系统总结还未见报 道, 尤其是对于近年来迅速发展起来的 COF 材料. 鉴于 该领域的重要性和快速发展, 在本综述中, 我们对氨基 酸功能化 MOFs 和 COFs 的最新研究进展进行综述, 重 点总结了以共价手段引入氨基酸的制备策略及其应用 (图 1). 对于 MOFs 而言, 功能化策略主要包括以氨基酸 或其衍生物作为配体, 通过合成后修饰共价连接氨基 酸, 或以氨基酸作为调节剂调控 MOFs 的生长过程; 氨 基酸功能化 COF 材料的制备主要通过合成后修饰的方 法引入特定氨基酸单元和序列. 在此基础上, 对这些材 料在手性拆分、催化、吸附和质子传导等领域的应用进 行了概述. 最后, 本综述分析了当前氨基酸功能化的晶 态多孔聚合物方向中面临的困难和挑战, 并对其未来研 究方向和应用前景进行了总结与展望.

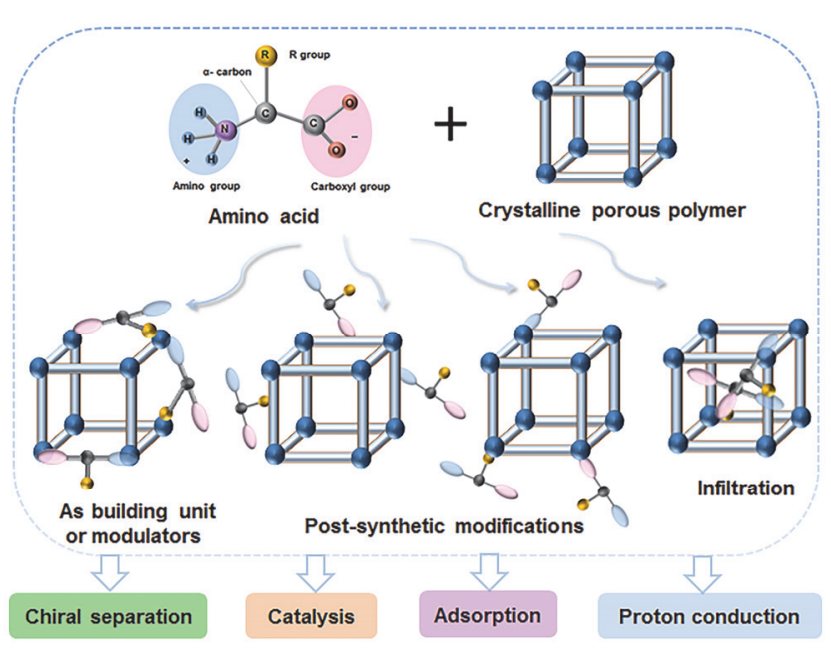

图 1 氨基酸功能化晶态多孔聚合物的制备策略.

Figure 1 Schematic illustration of synthetic strategies for amino acid functionalized crystalline porous polymer.

\section{2 氨基酸功能化 MOFs 的合成策略}

氨基酸种类繁多，其结构中不仅包含羧基和氨基， 侧链也具有丰富的官能团，可提供多种反应位点，通过 以下方式参与 MOFs 构筑: (1)羧基上的氧原子和氨基上 的氮原子与金属中心离子进行配位作用(图 2); (2)侧链 上的官能团，比如咪唑基、巯基等，与 MOFs 中的金属 中心进行配位; (3)氨基、羧基、侧链上的官能团还可用 于氨基酸的化学修饰或作为反应位点引入 $\mathrm{MOF}$ 骨架中. 此外, 氨基酸可以通过调节酸碱度、竞争配位作用, 调 控 MOFs 的成核和生长过程. 因此, 根据氨基酸的以上 特点和功能, 构筑氨基酸功能化 MOFs 的策略包括: (1) 氨基酸或其衍生物作为配体构筑 MOFs; (2)氨基酸通过 共价键或配位键合成后修饰 MOF 骨架; (3)氨基酸作为 调节剂调控 MOFs 的生长过程.

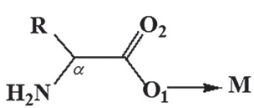

$\mu_{1}-\mathbf{O}_{1}$<smiles>[M]OC([M])OC(=O)C([R])N</smiles>

$\mu_{3}-\mathrm{O}_{1}: \mathrm{O}_{1} \mathrm{O}_{2}: \mathrm{O}_{2}$<smiles>[Y]C([Y13])C(OC)O[AlH]</smiles>

$\mu_{2}-\mathbf{O}_{1}: \mathbf{O}_{2}$

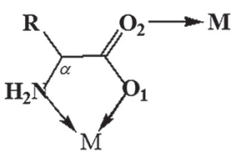

$\mu_{2}-\mathrm{N}_{1} \mathrm{O}_{1}: \mathrm{O}_{2}$

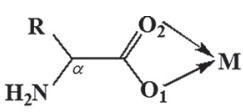

$\mu_{1}-\mathbf{O}_{1} \mathbf{O}_{2}$

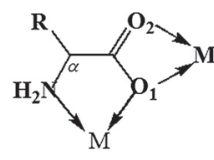

$\mu_{3}-\mathrm{N}_{1} \mathrm{O}_{1}: \mathrm{O}_{1} \mathrm{O}_{2}$
图 2 氨基酸与金属离子的潜在配位模型. 改编自文献[8b]

Figure 2 The potential coordination models of amino acids and metal ions. Adapted from the literature [8b]

\section{1 氨基酸或其衍生物作为配体构筑 MOFs}

氨基酸以羧基、氨基作为金属离子结合位点，通常 情况下倾向于通过 O、N-螯合模式构筑一维网络结构 ${ }^{[12]}$. Jacobson 等 ${ }^{[12]}$ 利用 $\mathrm{NiCl}_{2} \cdot 6 \mathrm{H}_{2} \mathrm{O} 、 L$-天冬氨酸三乙胺盐 $\left(\mathrm{Et}_{3} \mathrm{NH}\right)\left(L-\mathrm{H}_{2} \mathrm{Asp}\right)$ 和三乙胺 $\left(\mathrm{Et}_{3} \mathrm{~N}\right)$ 通过水热法合成了一 
种手性一维螺旋链杂化化合物 $\left[\mathrm{Ni}_{2} \mathrm{O}(L-\mathrm{Asp})\left(\mathrm{H}_{2} \mathrm{O}\right)_{2}\right]$ $4 \mathrm{H}_{2} \mathrm{O}$. 此一维结构可在高 $\mathrm{pH}$ 值反应条件下进一步通过 $\left(\mathrm{NiAsp}_{2}\right)^{2-}$ 桥连形成具有一维手性开放通道的三维框架 材料 $\left[\mathrm{Ni}_{2.5}-(\mathrm{OH})(L-\mathrm{Asp})_{2}\right] \cdot 6.55 \mathrm{H}_{2} \mathrm{O}$. 结构中的主要构筑 单元是由共边、共顶点 $\mathrm{Ni}(\mathrm{II})$ 八面体组成的一维螺旋链, 其中三聚体单元 $\mathrm{Ni}_{3}(\mathrm{OH}) \mathrm{O}_{12} \mathrm{~N}$ 通过 $\mathrm{Ni}(1) \mathrm{O}_{6}$ 八面体相互 连接, 每个天冬氨酸的氨基和羧基以 $\mu_{5}-\mathrm{N}_{1} \mathrm{O}_{1} \mathrm{O}_{4}$ : $\mathrm{O}_{1}: \mathrm{O}_{2}: \mathrm{O}_{3}: \mathrm{O}_{4}$ 的模式与五个 $\mathrm{Ni}^{2+}$ 配位, 螺旋链之间通过 $\left[\mathrm{NiAsp}_{2}\right]^{2-}$ 桥连形成一个三维框架. 在某些情况下, 氨 基酸中的 $\mathrm{O} 、 \mathrm{~N}$ 原子可以通过二齿、三齿桥连模式与金 属离子配位, 从而形成拓展的骨架结构. 例如, $L-$ 甘氨 酸( $L-$ Gly) 与 $\mathrm{Ni}^{2+} 、 \mathrm{Mn}^{2+}$ 或 $\mathrm{Co}^{2+}$ 的配位可以构建二维 $M O F s^{[13]}$, 其中 $L-G l y$ 上的羧基氧原子采用 $\mu_{2}-\mathrm{O}_{1}: \mathrm{O}_{2}$ 的配 位模式. $L$-苯丙氨酸 $(L-\mathrm{Phe})$ 或 $L$-谷氨酰胺 $(L-\mathrm{Gln})$ 与 $\mathrm{Cu}^{2+}$ 也可以构筑二维结构 ${ }^{[14]}$, 其中氨基酸配体以 $\mathrm{O} 、 \mathrm{~N}$-螯合 模式配位到金属离子上, 同时羧基中的另一个 $\mathrm{O}$ 原子也 与临近金属离子配位, 形成 $\mu_{2}-\mathrm{N}_{1} \mathrm{O}_{1}: \mathrm{O}_{2}$ 的配位模式.

使用纯氨基酸构筑三维 MOFs 的报道较少, 主要是 受到氨基酸的结构对称性较低的限制. 但若选择合适的 氨基酸在某些条件下也可以构筑三维结构, 例如, Park 等 ${ }^{[15]}$ 利用 $L$-谷氨酸 $(L-\mathrm{Glu}) 、 \mathrm{ZnSO}_{4} \cdot 7 \mathrm{H}_{2} \mathrm{O}$ 和 $\mathrm{NaOH}$ 在室 温水溶液中合成了一种水稳定性的三维结构 $\mathrm{ZnGlu}$ $\left[\mathrm{Zn}\left(\mathrm{H}_{2} \mathrm{O}\right)\left(\mathrm{C}_{5} \mathrm{H}_{7} \mathrm{NO}_{4}\right)\right] \cdot \mathrm{H}_{2} \mathrm{O}$. 该结构中, 锌离子与三个谷 氨酸中脱质子的羧基端以及一个水分子配位形成扭曲 的八面体构型. Serre 等 ${ }^{[16]}$ 报道了一种由 $L$-天冬氨酸作 为配体构筑的三维稳定结构 MIP-202(Zr), 该 MOF 材料 通过 $\mathrm{ZrCl}_{4}$ 与 $L$-天冬氨酸在水中反应得到, 其框架中 12 连接的 $\mathrm{Zr}_{6}\left(\mu_{3}-\mathrm{O}\right)_{4}\left(\mu_{3}-\mathrm{OH}\right)_{4}(\mathrm{COO}-)_{12}$ 团簇通过 $L$-天冬氨 酸桥连形成 fcu 拓扑学结构, 在强酸、强碱和沸水等条 件下均表现出良好的化学稳定性.
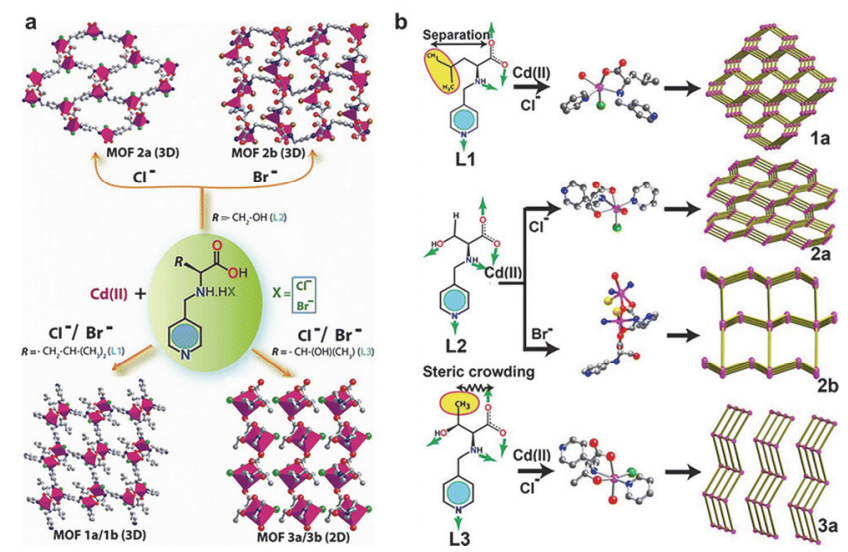

图 3 (a) 配体 L1、L2 和 L3 与 $\mathrm{Cd}(\mathrm{II})$ 反应生成相应的 MOF 结构(MOF $1 a / 1 b, 2 a / 2 b$ 和 $3 a / 3 b$ ) 示意图. (b) MOF $1 a, 2 a$ 和 $3 a$ 的次级结构单元和 拓扑简化模型. 改编自文献[17]

Figure 3 (a) Schematic representation of linkers L1, L2, and L3 that react with $\mathrm{Cd}(\mathrm{II})$ to produce corresponding MOF architectures (MOF $\mathbf{1 a} / \mathbf{1} \mathbf{b}, \mathbf{2 a} / \mathbf{2} \mathbf{b}$, and $\mathbf{3 a} / \mathbf{3 b}$ ). (b) MOFs 1a, 2a, and 3a with their SBUs and topological simplification model showing change in lattice arrangement. Adapted from the literature [17]
由于纯氨基酸易于形成低维的框架，为了构筑高孔 隙率的氨基酸 MOFs，化学修饰的氨基酸被用来合成三 维骨架结构. 2012 年, Banerjee 等 ${ }^{[17]}$ 报道六例氨基酸功 能化的手性 MOFs，作者利用吡啶修饰 $L$-亮氨酸 $(L-\mathrm{Leu}) 、 L-$ 丝氨酸 $(L-\mathrm{Ser}) 、 L$-苏氨酸 $(L-\mathrm{Thr})$, 再经过 $\mathrm{HCl}$ 或 $\mathrm{HBr}$ 处理, 设计并合成了含有 $\mathrm{Cl}^{-}$或 $\mathrm{Br}^{-}$的氨基酸衍生 物(L1、L2、 L3), 将其分别与 $\mathrm{Cd}\left(\mathrm{CH}_{3} \mathrm{COO}\right)_{2} \cdot 2 \mathrm{H}_{2} \mathrm{O}$ 进行 水热合成, 得到六例氨基酸功能化的 $\mathrm{MOF}$ 材料 $-\left[\mathrm{Cd}(\mathrm{L} 1)(\mathrm{Cl})\left(\mathrm{H}_{2} \mathrm{O}\right)\right] \infty(\mathbf{1 a}) 、 \quad[\mathrm{Cd}(\mathrm{L} 1)(\mathrm{Br})] \infty(\mathbf{1 b}) 、$ $\left.[\mathrm{Cd}(\mathrm{L} 2)(\mathrm{Cl})]\left(\mathrm{H}_{2} \mathrm{O}\right)\right] \infty(\mathbf{2 a}) 、\left[\mathrm{Cd}_{2}(\mathrm{~L} 2)_{2}(\mathrm{Br})_{2}\left(\mathrm{H}_{2} \mathrm{O}\right)_{3}\right] \infty(\mathbf{2 b}) 、$ $\left[\mathrm{Cd}(\mathrm{L} 3)(\mathrm{Cl})\left(\mathrm{H}_{2} \mathrm{O}\right)_{2}\right] \propto(\mathbf{3 a})$ 和 $\left[\mathrm{Cd}(\mathrm{L} 3)(\mathrm{Br})\left(\mathrm{H}_{2} \mathrm{O}\right)_{2}\right] \propto(\mathbf{3 b})($ 图 $3 a \sim 3 b)$. 其中结构 $1 \mathbf{a} 、 \mathbf{1 b} 、 \mathbf{2 a}$ 和 $\mathbf{2 b}$ 为三维骨架, 结构 $3 \mathbf{a}$ 和 $3 \mathbf{b}$ 为二维骨架, 结构分析显示这是由于后者框架 中的卤素原子特殊的位置阻碍了结构的进一步拓展. 1a 中 $\mathrm{Cd}(\mathrm{II})$ 与 $\mathrm{L} 1$ 单体上的氨基氮原子、吡啶基氮原子、 羧基氧原子以及氯离子配位, 形成一个扭曲的四方锥构 型，其中氨基氮原子与 $\mathrm{Cd}(\mathrm{II})$ 配位形成了一个手性中心, 双金属 $\mathrm{Cd}(\mathrm{II})$ 中心之间通过羧基氧原子桥连形成一维螺 旋形孔道.

Turner 等 ${ }^{[18]}$ 报道了利用菜二酰亚胺(NDI)修饰的 $L-$ 丙氨酸 $(L-\mathrm{Ala}) 、 L$-亮氨酸、 $L$-苯丙氨酸三种氨基酸配体, 分别与 $\mathrm{s}$-区和 $\mathrm{d}-$ 区的金属离子 $\mathrm{Mn}^{2+} 、 \mathrm{Cd}^{2+}$ 配位合成的 三维结构. 李晖课题组 ${ }^{[19]}$ 通过控制反应温度、金属盐、 溶剂等条件, 由 NDI 修饰的氨基酸配体与 $\mathrm{f}-$ 区金属离子 配位合成了三维稀土配位聚合物. 郑发鲲课题组 ${ }^{[20]}$ 设 计并合成了四种氨基酸功能化的配体, $(S) /(R)-N-[2-(1 H-$ 四唑-5-基)-乙基]-色氨酸( $\mathrm{H}_{2}$ tzet $) 、(S) /(R)-N$-[2-(1H-四唑5 -基)-乙基]-脯氨酸( $\mathrm{H}_{2}$ tzep), 分别与 $\mathrm{ZnCl}_{2} 、 \mathrm{NaN}_{3}$ 在水相 中原位合成四例 $D / L$-脯氨酸 $(D / L-P r o) 、 D / L$-色氨酸 $(D / L-S e r)$ 功能化的二维材料一 $[\mathrm{Zn}(\text { tzet })]_{n}(\mathbf{4 a}$ 和 $\mathbf{4 b})$ 和 $\left[\mathrm{Zn}(\right.$ tzep $\left.)\left(\mathrm{H}_{2} \mathrm{O}\right)_{2}\right] \cdot \mathrm{H}_{2} \mathrm{O}(\mathbf{5 a}$ 和 $\mathbf{5 b})$. 单晶结构分析显示在 $\mathbf{4 a}$ 和 $4 \mathbf{b}$ 结构中, 每个 $\mathrm{Zn}^{2+}$ 与来自三个配体的两个 $\mathrm{N}$ 原 子和两个 $\mathrm{O}$ 原子配位形成一个四方棱雉构型, 每个配体 的羧基氧原子与三个 $\mathrm{Zn}^{2+}$ 连接，四唑负离子充当一个 三齿配体，羧基基团桥连两个 $\mathrm{Zn}^{2+}$ 形成一个螺旋链，相 邻的链通过 $\mu_{2}-\kappa \mathrm{N} 1: \kappa \mathrm{N} 4$ 模式桥连四唑环形成一个二维 的纳米片结构. $5 \mathbf{a}$ 和 $\mathbf{5 b}$ 均由 $\mathrm{Zn}^{2+}$ 以五配位模式与一个 配体上的一个 $\mathrm{O}$ 原子、两个 $\mathrm{N}$ 原子以及两个水分子连 接形成扭曲的三角双雉构型(图 4a).

此外，还可通过引入多齿有机配体来弥补氨基酸配 体的低对称性的问题. 这种利用混合配体策略来制备三 维氨基酸 MOFs, 在一定程度上丰富了框架结构的种类. 2011 年, 张健课题组 ${ }^{[21]}$ 报道了两例利用 $D / L$ - 丝氨酸与 对苯二甲酸(bdc)和 $\mathrm{Zn}\left(\mathrm{NO}_{3}\right)_{2} \cdot 6 \mathrm{H}_{2} \mathrm{O}$ 水热合成的丝氨酸 功能化的手性三维 $\mathrm{MOFs}-\left[\mathrm{Zn}_{4}(\mathrm{bdc})_{2}(L-\mathrm{Ser})_{2}\right] \cdot$ $36 \mathrm{DMF}(1-\mathrm{L})$ 和 $\left[\mathrm{Zn}_{4}(\mathrm{bdc})_{2}(D-\mathrm{Ser})_{2}\right] \cdot 36 \mathrm{DMF}(1-\mathrm{D})$. 该课题 组 ${ }^{[22]}$ 又报道了四例利用 $D / L$-丙氨酸、 $L$ - 丝氨酸、 $L$-缬氨 酸 $(L-V a l)$ 分别与 $\mathrm{Zn}\left(\mathrm{NO}_{3}\right)_{2} \cdot 6 \mathrm{H}_{2} \mathrm{O} 、 5$-甲基四氮唑合成三 维手性 ZMOFs(图 4b). 其中以丙氨酸构筑的三维 MOFs 


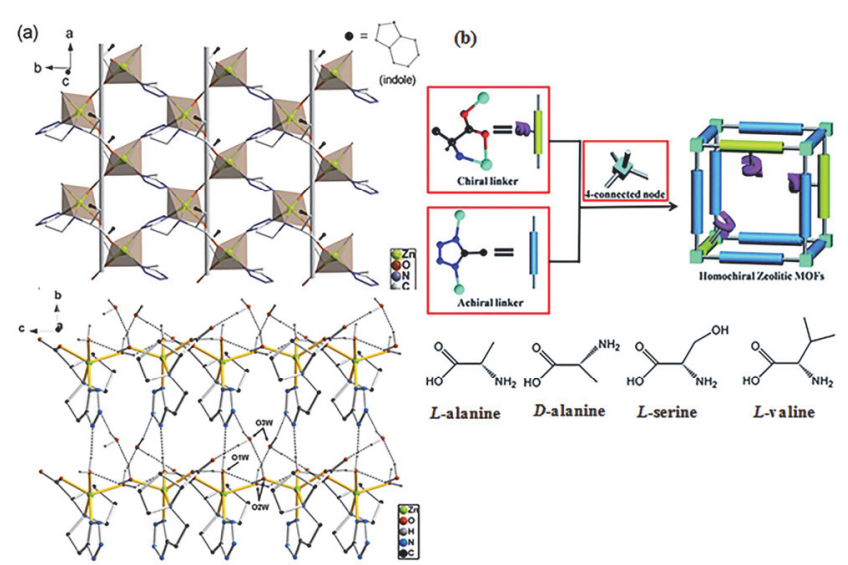

图 4 (a) $[\mathrm{Zn}(\mathrm{tzet})]_{n}(\mathbf{4 b})$ 和 $\left[\mathrm{Zn}(\mathrm{tzep})\left(\mathrm{H}_{2} \mathrm{O}\right)_{2}\right] \cdot \mathrm{H}_{2} \mathrm{O}$ (5b)的二维结构. (b) 手性 ZMOFs 的合成策略. 改编自文献[20], [22]

Figure 4 (a) Structure of 2D network in $\mathbf{4 b}$ and $5 \mathbf{b}$. (b) The synthesis strategy of the homochiral ZMOFs. Adapted from the literature [20], [22]

为例，该晶体的不对称单元包含四个 $\mathrm{Zn}(\mathrm{II})$ 、六个 5-甲 基四氮唑 (5-Hmtz)、两个丙氨酸以及两个有序的 DMF 分子组成. 晶体结构中存在两种类型的 $Z n(I I)$ 中心, 第 一类 Zn(II)中心与三个 5-甲基四氮唑的三个氮原子和一 个丙氨酸的羧基氧原子配位形成四面体构型, 而另一类 $\mathrm{Zn}(\mathrm{II})$ 中心则与两个 5 -甲基四氮唑的两个氮原子和一个 丙氨酸的氨基氮原子配位形成扭曲三角双雉构型. $\mathrm{Zn}(\mathrm{II})$ 中心通过 5-甲基四氮唑桥连形成层状结构, 丙氨 酸进一步连接相邻的层形成三维框架. 王博课题组 ${ }^{[23]}$ 利用 2 -甲基咪唑 $(\mathrm{mIm})$ 与 $D$-组氨酸 $(D$-His $)$ 为配体, 与 $\mathrm{Zn}\left(\mathrm{NO}_{3}\right)_{2} \bullet 4 \mathrm{H}_{2} \mathrm{O}$ 一锅法合成了手性 $\mathrm{MOF}$ 材料一 $D$-his-ZIF-8, 其中氨基酸原位取代 ZIF-8 骨架中部分 2甲基咪唑, 使其具有手性性质. Kaskel 等 ${ }^{[24]}$ 利用联苯二 甲酸修饰的带氨基保护基的脯氨酸 (Boc-Pro) 与 4,4',4'-[苯-1,3,5-三(羰基亚氨基)]三苯甲酸酯 $\left(\mathrm{H}_{3} \mathrm{btctb}\right.$ )、 $\mathrm{Zn}\left(\mathrm{NO}_{3}\right)_{2} \bullet 4 \mathrm{H}_{2} \mathrm{O}$ 原位合成了脯氨酸功能化的三维的手性 介孔 MOF 材料一DUT-32-NHProBoc, 单晶结构表征显 示该结构包含四种不同尺寸的孔道. 但在加热脱 Boc保护基的过程中, 该材料的手性会丧失.

氨基酸可以通过脱水缩合反应合成多肽, 改变氨基 酸的种类和排列顺序可以得到成千上万种多肽. 多肽具 有更加多样和灵活的金属配位模式, 因而可用于构筑 $2 \mathrm{D}$ 或 $3 \mathrm{D}$ 多肽基 $\mathrm{MOF}^{[8]}$. Rosseinsky 等 ${ }^{[25]}$ 选用具有化学 多样性和复杂空间构象的三肽一甘氨酸-甘氨酸 $-L$-组氨 酸(Gly-Gly-L-His, GGH)作为 MOF 材料的连接单元, 与 $\mathrm{Zn}(\mathrm{OAc})_{2} \cdot 2 \mathrm{H}_{2} \mathrm{O}$ 共同构建了新型柔性 $\mathrm{MOF}$ 材料 - ZnGGH(图 5a). 如图 5b 所示, 双重脱质子的三肽可 以通过四个位点(氨基氮原子、羧基氧原子、咪唑单元 中两个氮原子)与四个金属中心配位, 每个 $Z n(I I)$ 中心可 与四个多肽单元配位形成四面体构型. 结构表征显示该 材料具有一维孔道, 结构中包含刚性的 Zn-His 层, 层间 通过构象灵活的 Gly-Gly 链连接, 赋予整个框架结构一
定的柔性，单晶结构分析显示该晶体结构可响应化学环 境的改变.

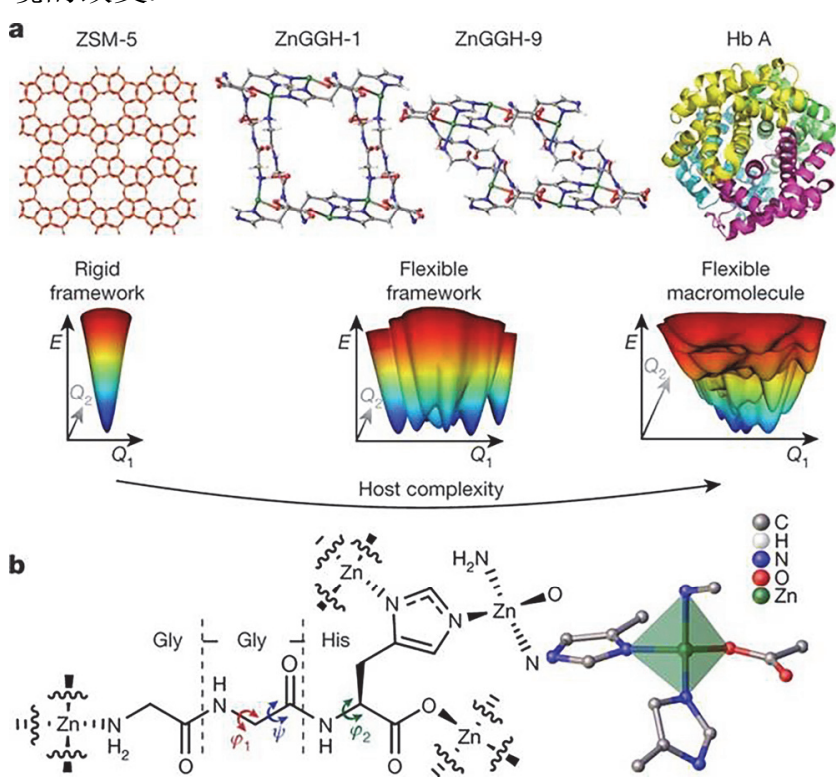

图 $5 \mathrm{ZnGGH}$ 在构象能量图中的结构灵活性. (a) ZSM-5 是一种刚性 的多孔框架, 新型 $\mathrm{ZnGGH}$ 材料是一类柔性的多孔框架. 与蛋白质的 大分子状态不同, $\mathrm{ZnGGH}$ 是晶态固体框架材料, 限制了配体有效的构 象空间. 本图中的能量图是假设的示意图, 用以反映对应材料的结构 性质. (b) 双重脱质子的三肽 Gly-Gly- $L$-His 作为四爪配体形成 $\mathrm{ZnGGH}$ 框架. 改编自文献[25]

Figure 5 The structural flexibility of $\mathrm{ZnGGH}$ in the context of a conformational energy landscape. (a) ZSM-5 is a rigid porous framework, the new $\mathrm{ZnGGH}$ compound reported here is a flexible porous framework. In contrast to the macromolecular state of proteins, ZnGGH is a crystalline solid framework, which restricts the available conformational space of the linker. All energy landscapes drawn in this figure are hypothetical illustrations that reflect the structural properties of the corresponding materials. (b) The doubly deprotonated tripeptide Gly-Gly- $L$-His acts as a tetratopic linker to form the $\mathrm{ZnGGH}$ framework. Adapted from the literature [25]

\section{2 氨基酸合成后修饰共价连接 MOF 骨架}

由于氨基酸结构的柔性和配位模式的多样性，以氨 基酸或其衍生物作为配体构筑 MOFs, 很难实现结构的 准确预判与精准调控. 通过合成后修饰策略在 MOF 骨 架中化学修饰引入氨基酸单元, 可以实现氨基酸空间排 布和修饰程度的调控, 进而对材料的物理、化学性质进 行调节 ${ }^{[26]}$.

通过合成后修饰策略引入氨基酸多是借助固态多 相合成的方法, 利用氨基酸中的羧基与氨基官能化的 MOFs 之间的酰胺化反应, 在合成过程中往往需要加入 偶联剂提高反应活性 ${ }^{[27]}$. 此外, 为了避免副反应的发 生, 通常还需要对氨基酸的氨基端进行保护. 2011 年, Canivet 课题组 ${ }^{[28]}$ 利用芴甲氧羰基(Fmoc)保护的氨基酸 Fmoc-Pro-OH 和 Fmoc-Ala-OH 对(In)MIL-68- $\mathrm{NH}_{2}$ 进行 修饰，在三吡咯烷基溴化鏻六氟磷酸盐(PyBroP)和 4-二 甲氨基吡啶(DMAP)偶联剂的共同作用下，通过加热引 发耦合反应以及氨基脱保护，成功合成两例氨基酸功能 化的手性 MOFs. 相对于 MIL-68- $\mathrm{NH}_{2}$ 的微孔结构, 偶联 
剂 PyBroP 和保护基 Fmoc 尺寸较大, 在空间上阻碍了氨 基酸的接枝能力, 产率仅为 $10 \%$. 为提高反应的产率和 产物的纯度, Canivet 课题组 ${ }^{[29]}$ 首次提出利用微波辅助 加热的方法在 MOF 骨架上共价接枝氨基酸和寡肽, 通 过优化偶联剂、碱、溶剂等反应条件, 以快速、高效的 方式在 Al-MIL-101- $\mathrm{NH}_{2}$ 、In-MIL-68- $\mathrm{NH}_{2} 、 \mathrm{Zr}-\mathrm{UiO}-66-$ $\mathrm{NH}_{2}$ 三种 MOFs 上成功修饰手性位点(图 6a). 其中 Al-MIL-101- $\mathrm{NH}_{2}$ 在 $20 \mathrm{~min}$ 即可达到 15\%的 HO-Pro 接 枝率，并在 $30 \mathrm{~min}$ 达到 $60 \%$ 的 HO-Gly-Pro 接枝率. 微 波法不仅加快了耦合过程, 而且在脱去叔丁氧羰基 (Boc)保护基的过程中保证了材料的接枝率和骨架的完 整性. 2016 年, Yaghi 课题组 ${ }^{[30]}$ 利用七步串联反应引入氨 基酸序列修饰多组分 MTV-IRMOF-74-III, 合成了多肽 功能化类酶 MOF 材料. 作者先选用 $-\mathrm{CH}_{3}$ 和 $-\mathrm{CH}_{2} \mathrm{NHBoc}$ 预功能化有机配 体构筑系列 $\mathrm{MOF}$ 材料一 $\operatorname{MTV}-\left(\mathrm{CH}_{3}\right)_{(1-x)}\left(\mathrm{CH}_{2} \mathrm{NHBoc}\right)_{x}(x=0.2 、 0.4 、 0.6$ 和 0.8$)$, 然 后利用 $N$-(叔丁氧基)- $L$-丙氨酸(Boc- $L$-Ala)对其修饰, 发 现当 $x=0.4$ 可以保证保护基的定量脱去, 并且与 $L$-丙氨 酸 (L-Ala) 生成肽键的产率达到 $97 \%$, 因此选用 MTV- $\left(\mathrm{CH}_{3}\right)_{0.6}-\left(\mathrm{CH}_{2} \mathrm{NH}_{2}\right)_{0.4}$ 作为起始反应物, 在微波辅助 加热下通过连续反应将氨基酸有序引入, 得到三肽修饰 的材料 MTV- $\left(\mathrm{CH}_{3}\right)_{0.6}\left(\mathrm{CH}_{2} \mathrm{NH}-\mathrm{Ala}-\mathrm{Gly}-\mathrm{Pro}-\mathrm{NH}_{2}\right)_{0.2}$ 和 MTV- $\left(\mathrm{CH}_{3}\right)_{0.6}\left(\mathrm{CH}_{2} \mathrm{NH}-\mathrm{Asp}-\mathrm{His}-\mathrm{Cys}-\mathrm{NH}_{2}\right)_{0.1}$.

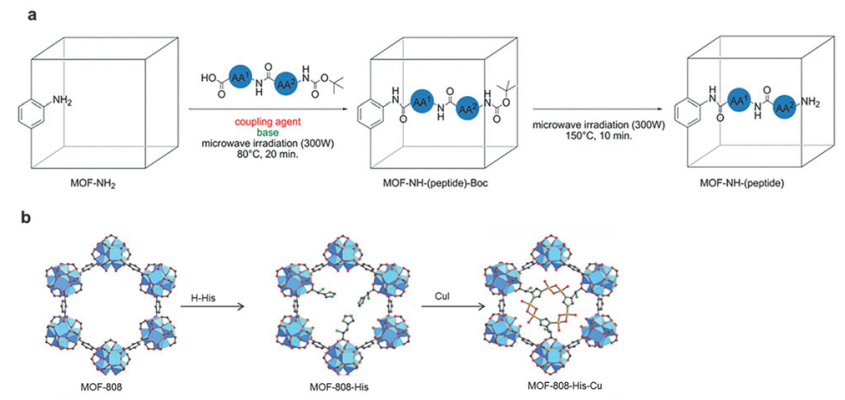

图 6 (a) 在多种 MOFs 中进行两步多肽接枝过程. (b) 通过将 MOF-808 中甲酸盐替换为含咪唑单元配体和 $\mathrm{Cu}^{(\mathrm{I})}$ 金属化制备催化剂. 改编自文献[29], [31]

Figure 6 (a) The two-step peptide grafting process into various MOFs. (b) Synthesis of the catalysts comprising the replacement of formate with imidazole-containing ligands and metalation with $\mathrm{Cu}^{(\mathrm{I})}$. Adapted from the literature [29], [31]

除了借助 MOFs 中的氨基进行酰胺化反应, 还可利 用 MOF 骨架中金属位点与氨基酸结构中的 $\mathrm{O} 、 \mathrm{~N}$ 原子 的配位作用实现功能化, 这种策略避免了偶联剂以及保 护基的使用，降低了反应条件的复杂性. 2018 年, Yaghi 课题组 ${ }^{[31]}$ 报道了在 MOF-808 的金属团簇上修饰含咪唑 单元的有机配体, 在此基础上进行金属化构筑仿酶活性 位点 (图 6b). MOF-808 的 $\mathrm{Zr}_{6}$ 团簇上除了连接均苯三酸 (BTC)配体外, 还配有甲酸根、水和氢氧根, 含有羧基的 配体(如 $L$-组氨酸)可以通过替换这些组分配位到金属团 簇, 再通过与碘化亚铜的反应进行后金属化, 实现活性 中心的构筑. 由此制备的催化剂 MOF-808-His-Cu 可以
将甲烷选择性氧化为甲醇.

\section{3 氨基酸作为调节剂调控 MOFs 的生长过程}

氨基酸也可以作为 MOFs 合成过程中的竞争调节 剂、添加剂等，通过竞争配位、调节酸碱度等方式调控 MOFs 成核和生长过程，手性氨基酸还可诱导骨架中形 成手性环境.

通常认为在 MOFs 合成中加入 “单边羧基” 的竞争 调节剂, 如苯甲酸、乙酸、甲酸等, 可以调控结晶的动 力学过程, 通过竞争配位促进反应的可逆性以提高结晶 度. Forgan 课题组 ${ }^{[32]}$ 报道了利用氨基酸作为调节剂调控 具有不同长度的配体(L6、L7、L8)的 Zr-MOFs 和 Hf-MOFs 的生长(L6: 联苯二甲酸; L7: 偶氮苯-4,4-二羧 酸; L8: 4,4-二苯乙烯二羧酸)(图 7a). 他们分别选取 $D L-$ 苏氨酸 $(D L-\mathrm{Thr}) 、 L$-精氨酸 $(L-\mathrm{Arg}) 、 L$-色氨酸、 $L$ - 亮氨 酸、 $L$-丙氨酸、 $L$-脯氨酸 $(L-P r o)$ 与 $\mathrm{HCl}$ 共同作为调节剂, 发现加入 $L$-脯氨酸和少量 $\mathrm{HCl}$ 可以成功合成无缺陷的 Zr-MOF 和 Hf-MOF 单晶，与使用其他常用的调节剂相 比，氨基酸调节剂的用量明显减少.

在某些情况下，竞争剂的加入也会在 MOFs 合成过 程中引发缺陷位点. Shafir 课题组 ${ }^{[33]}$ 选用了 $L$-脯氨酸、 $L-$ 甘氨酸、 $L$-苯丙氨酸三种氨基酸来调节 Z r-MOFs 的生 长, 不仅实现了晶体大小的调控, 而且还能将氨基酸结 合在缺陷位点, 实现骨架功能化 (图 7b). 通过控制反应 条件和配体结构, 氨基酸与有机配体比例最高可到 1 : 1, 由此制备氨基酸功能化 MOF 材料. 2019 年，赵钟兴 课题组 ${ }^{[34]}$ 利用分子印迹策略, 选用 $L$-脯氨酸、 $L$-苯丙氨 酸、 $L$-色氨酸 $(L-\operatorname{Trp})$ 三种疏水性的氨基酸在 UiO-66 合 成过程中原位构筑具有印迹的缺陷, 并成功将其孔道从 微孔扩大至介孔(3.1 nm). 在这一体系中, $L$-脯氨酸未能 成功引发缺陷, 猜测这与反应体系的化学环境、氨基酸 自身性质等有关.

Falcaro 及其合作者 ${ }^{[35]}$ 提出仿生矿化策略，即利用 生物分子调控 MOF 材料的形貌，他们借助 20 种天然氨 基酸作为 ZIF-8 生长过程中的晶体和结构导向剂, 发现 氨基酸侧链的极性、亲疏水性、带电荷性等对于 ZIF-8 的形貌、尺寸、颗粒数都有显著影响. 其中加入 $L$-赖氨 酸 $(L-\mathrm{Lys}) 、 L$-组氨酸、 $L$-酪氨酸 $(L-\mathrm{Tyr}) 、 L$-精氨酸的反 应液中均没有生成 ZIF-8, 作者认为这是由于 Lys、His 和 Arg 的正电性与 $\mathrm{Zn}^{2+}$ 相斥, 阻碍了 $\mathrm{ZIF}-8$ 晶体的生长, 而 Tyr 由于在水中的溶解度很低, 不能有效地参与结晶 过程，其它氨基酸的加入则使得 ZIF-8 晶体具有独特的 大小和形态. 基于氨基酸固有的手性性质，Zaworotko 等 ${ }^{[36]}$ 在具有高度对称结构的 MOF-5 的合成过程中分别 加入 $L$-脯氨酸和 $D$-脯氨酸, 在氨基酸的手性诱导作用 下得到了具有手性结构的 $\Lambda$-CMOF-5 和 $\Delta-C M O F-5$ (图 7c).

总的来说，面向不同的应用需求，以上三种制备策 略各具特点，但是也存在各自的局限性. 通过氨基酸及 

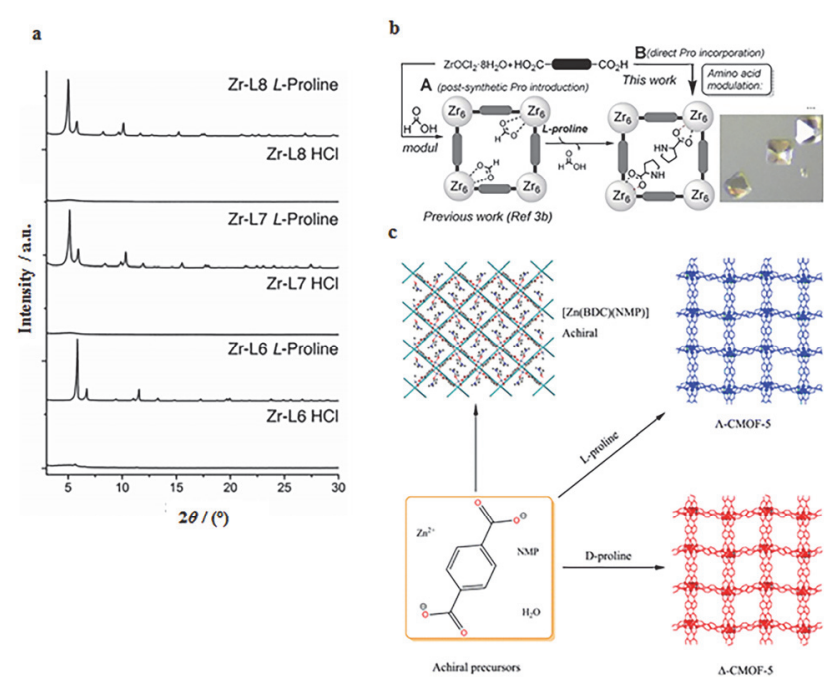

图 7 (a) 水热法合成锆基 MOF 过程中加入 5 equiv. $L$-脯氨酸、 1 equiv. $\mathrm{HCl}$ 以及单独加入 $\mathrm{HCl}$ 所得产物的 PXRD 图谱比较. (b) $L$-脯氨酸固定 在 Zr-MOF 上以及氨基酸调节 MOFs 的合成; (c) CMOF-5 的合成. $L / D$ 脯氨酸添加剂的存在诱导产生手性, 手性 CMOF-5 晶体得以生长和分 离. 不加入脯氨酸的情况下, 得到非手性的正方形网格 [ $\mathrm{Zn}(\mathrm{BDC})-$ (NMP)]. 改编自文献[32], [33], [36]

Figure 7 (a) PXRD patterns showing the effects of incorporation of 5 equivalents of $L$-proline and one of $\mathrm{HCl}$ into solvothermal syntheses of $\mathrm{Zr}$ MOFs of $\mathrm{L} 6 \sim \mathrm{L} 8$ compared to addition of $\mathrm{HCl}$ alone. (b) Anchoring of $L$-proline onto a $\mathrm{Zr}$ MOF and amino acid-modulated MOF synthesis. (c) Synthesis of CMOF-5. The presence of $L$ - or $D$-proline additives induces chirality and enables the growth and isolation of CMOF-5 crystals. An achiral square grid net, [Zn(BDC)(NMP)], forms in the absence of proline. Adapted from the literature [32], [33], [36]

其衍生物构筑 MOFs 是最直接的策略, 可以保证氨基酸 在骨架中的比例, 同时还可引入柔性特征、手性环境等; 但是由于氨基酸结构的柔性, 直接构筑骨架具有一定的 难度, 且较难实现对最终结构的预测与控制. 将氨基酸 共价修饰到结构明确的骨架上, 可以避免上述提到的问 题, 且该方法具有较好的普适性; 但同时存在着修饰程 度难以准确调控、氨基酸堵塞孔道、脱保护过程中丧失 手性等问题. 以氨基酸作为调节剂的方法, 主要是用于 MOF 晶体的形貌、结晶度、尺寸、缺陷等的调控, 某些 条件下也可以诱导框架手性中心的形成, 因此制备得到 的MOFs 中氨基酸负载量较低、甚至几乎没有. 因此, 可 根据不同的应用选择合适的制备策略, 以及在此基础上 探究新方法.

\section{3 氨基酸功能化 COFs 的合成策略}

与氨基酸功能化 MOFs 相比, 在 COFs 体系中引入 氨基酸的相关研究相对较少, 主要包括物理吸附和共价 修饰两种方法 ${ }^{[37]}$. 通过物理吸附作用将氨基酸负载在 COFs 颗粒表面或者或孔道内, 其负载效率受氨基酸分 子与 $\mathrm{COFs}$ 之间的亲和力的强弱以及 $\mathrm{COFs}$ 孔径与氨基 酸分子尺寸的匹配程度的影响. 这种非共价吸附作用得 到的氨基酸 COF 材料的结构一般稳定性不好, 容易发 生氨基酸的脱落, 在实际应用过程中可能会影响其性
能. 另一种更为常用的策略，即通过合成后修饰实现 $\mathrm{COF}$ 骨架氨基酸功能化, 可以有效避免氨基酸的脱落 并提高修饰程度.

2018 年, 张振杰及其合作者 ${ }^{[38]}$ 通过共价耦合策略, 利用 $\mathrm{COF}$ 骨架上的羧基和生物分子中氨基之间的脱水 缩合反应，成功在 $\mathrm{COF}$ 结构上修饰氨基酸、多肽、酶. 他 们首先合成了酰亚胺连接的介孔 $\mathrm{COF}$ 材料一 $\mathrm{COF} 1$, 通 过甲苯胺蓝染色实验和酸碱滴定实验证实该 $\mathrm{COFs}$ 含有 约 5\%的- $\mathrm{COOH}$ 残基. 在 $\mathrm{pH}$ 为 7 的 2-( $N$-吗啉)乙磺酸缓 冲溶液中, 将 $L$-赖氨酸或三肽赖氨酸-烦氨酸-苯丙氨酸 (Lys-Val-Phe)与 COF1 混合反应, 并加入偶联剂 1-(3-二 甲氨基丙基)-3-乙基碳二亚胺(EDC)和 $N$-着基硫代琥珀 酰亚胺(NHS), 完成生物分子的共价修饰. 表征结果显 示, 与物理吸附方法修饰的功能化 COFs 相比, 通过共 价修饰的 COFs 中氨基酸负载率更高、浸出率则更低, 由此说明 COFs 与生物分子间形成了强相互作用.

蓝芳课题组 ${ }^{[39]}$ 报道了谷胱甘肽修饰的磁性 COF 核 壳结构微球. 首先通过 $\mathrm{FeCl}_{3} \cdot 6 \mathrm{H}_{2} \mathrm{O} 、 \mathrm{Na}_{3} \mathrm{Cit} \cdot 2 \mathrm{H}_{2} \mathrm{O}$ 、 $\mathrm{CH}_{3} \mathrm{COONa}$ 在溶剂热条件下合成了磁性纳米晶体簇 $\mathrm{MCNCs}$, 然后加入 $\mathrm{COF}$ 单体 1,3,5-三氨基苯基苯、2,5二乙烯基对苯二甲醛, 合成了以 $\mathrm{MCNCs}$ 为核、 $\mathrm{COFs}$ 为壳的 MCNC@COFs(图 8a), 再通过点击反应在 COF 层修饰高度亲水性的谷胱甘肽, 由此制备的微球可用于 糖肽的选择性识别. 张新祥课题组 ${ }^{[40]}$ 通过两步合成后 修饰借助银纳米颗粒在 COFs 上修饰谷胱甘肽. 选用 $\mathrm{TpPa}-1$ 作为基底均匀负载银纳米颗粒，谷胱甘肽结构 中两亲性的官能团使其可通过自组装结合到银纳米粒 子表面，由此制备的功能化材料中谷胱甘肽和银纳米颗 粒都可以提供丰富的结合位点用于糖肽的富集.

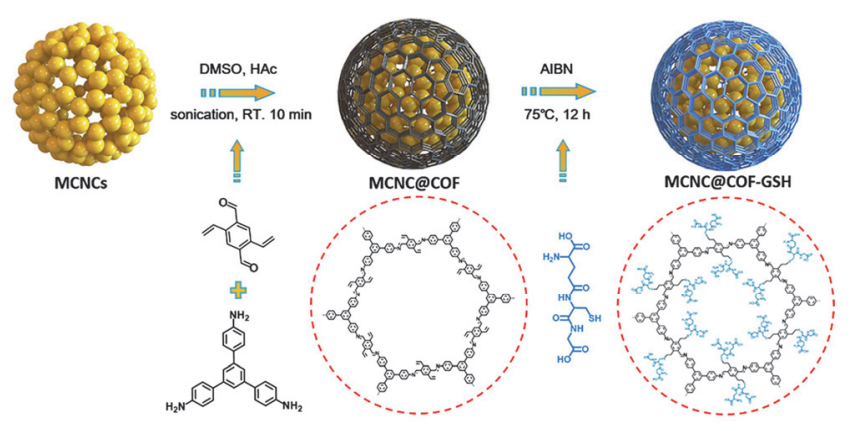

图 8 磁性核壳结构 MCNC@COF@GSH 的合成路径示意图. 改编自 文献[39]

Figure 8 Schematic representation of the synthetic route of the MCNC@COF@GSH microspheres. Adapted from the literature [39]

\section{4 应用}

晶态多孔聚合物骨架的氨基酸功能化, 可以实现孔 道尺寸和环境的调节, 进而调控骨架与客体分子的相互 作用; 还可赋予骨架一定的柔性, 响应外界刺激表现出 骨架结构的变化, 实现选择性吸附与识别. 手性氨基酸 的引入，可以在骨架中构筑手性环境、手性催化活性位 
点, 用于手性拆分和不对称催化. 此外, 通过氨基酸及 其序列的修饰, 可以借助框架材料构筑仿酶位点, 实现 特异性识别和催化. 氨基酸的引入, 赋予了晶态多孔聚 合物丰富的结构特征和功能，在众多领域中表现出巨大 的应用前景. 在这一部分, 我们将主要介绍氨基酸功能 化 MOFs、COFs 在手性拆分、吸附、催化、质子传导 等方面的应用(表 1).

\section{1 手性拆分}

手性拆分技术是在手性助剂的作用下将外消旋体 化合物拆分成为纯对映体化合物，在医药、工业等领域 均具有重要的意义 ${ }^{[41]}$. 与传统多孔材料分子篮、活性炭 等相比, MOF 材料更易功能化, 可形成高度有序的手性 孔道, 并且可以通过调控孔道结构、优化传质过程和手 性识别位点, 在手性分离方面表现出更大的优势 ${ }^{[42]}$. 基 于氨基酸固有的手性以及丰富的侧链基团, 可以从分子 水平上设计并有效调控 MOFs 的手性环境, 有望实现优 异的手性拆分效果, 这里主要介绍 MOF 材料在色谱拆 分技术、萃取拆分技术、膜手性拆分技术中的应用.

以手性固定相(CSP)法为代表的色谱拆分技术，具 有分离时间短、手性选择性高等优点; 将氨基酸功能化 的手性 MOFs 用作高效液相色谱、气相色谱的固定相可 以实现手性分离. 袁黎明课题组 ${ }^{[43]}$ 报道了 6 种基于氨基 酸的手性 MOFs, 分别为 $[\mathrm{Zn}(L \text {-tyr })]_{n}(L$-tyrZn) 、 $\left[\mathrm{Zn}_{4}(\mathrm{btc})_{2}(\mathrm{Hbtc})(L-\mathrm{His})_{2}\left(\mathrm{H}_{2} \mathrm{O}\right)_{4}\right] \cdot 1.5 \mathrm{H}_{2} \mathrm{O}$ 、 $\quad\left[\mathrm{Zn}_{2}(L-\operatorname{trp})_{2}-\right.$ (bpe $\left.)_{2}\left(\mathrm{H}_{2} \mathrm{O}\right)_{2}\right] \cdot 2 \mathrm{H}_{2} \mathrm{O} \cdot 2 \mathrm{NO}_{3} \quad 、 \quad\left[\mathrm{Co}_{2}(L-\mathrm{Trp})(\mathrm{INT})_{2}\left(\mathrm{H}_{2} \mathrm{O}\right)_{2}-\right.$ $\left.\left(\mathrm{ClO}_{4}\right)\right]$ 、 $\left[\mathrm{Co}_{2}(\mathrm{sdba})\left((L-\mathrm{Trp})_{2}\right]\right.$ 和 $\left[\mathrm{Co}(L-\mathrm{Glu})\left(\mathrm{H}_{2} \mathrm{O}\right) \cdot \mathrm{H}_{2} \mathrm{O}\right]$, (其中 INT: 碘硝基四唑; bpe: 双酚 A 聚氧乙烯醚; $\mathrm{H}_{3}$ btc:
均苯三甲酸), 表征分析结果表明它们均具有手性性质, 将其用于高效液相色谱的手性固定相可选择性分离 1,2联苯-1,2-乙二醇外消旋体等物质. 2018 年，严秀平课题 组 ${ }^{[44]}$ 利用合成后修饰法在 $\mathrm{NH}_{2}$-MIL-101 引入不同的手 性识别位点, 制备系列手性 MOFs 用于毛细管色谱柱的 涂层. 其中 $L$-脯氨酸修饰制备的 MIL-101- $L$-Pro, 其孔 道中 $L$-脯氨酸可提供手性位点和氢键作用, 然而受孔道 中较大尺寸的 Boc 保护基的影响, 其手性分离效果不 佳.

MOFs 作为手性固定相易受稳定性差、颗粒非均质 性等限制，进而导致保留时间长、色谱性能差、分离能 耗高等问题. 与手性固定相相比，固相萃取技术可以通 过移除干扰物、提高痕量浓度或样品纯化等，实现更为 有效的分离. Martí-Gastaldo 等 ${ }^{[45]}$ 报道了一种基于三肽 (Gly- $L-H i s-G l y)$ 的手性三维铜基 MOF 材料- $\mathrm{Cu}(\mathrm{GHG})$, 结构分析表明该框架的一维贯穿孔道中包含多种官能 团如羧基、酰胺基、氨基和咪唑基，并且孔径尺寸大于 $2 \mathrm{~nm}$, 能够允许客体分子顺利扩散进入该手性孔道 (图 $9 \mathrm{a} \sim 9 \mathrm{c})$. 将 $\mathrm{Cu}(\mathrm{GHG})$ 作为固相萃取吸附剂分离手性药 物脱氧麻黄碱(MA)、麻黄碱(EP)的外消旋体混合物, 能 够在 $4 \mathrm{~min}$ 内分离出大于 $50 \%$ 的 $(+)$-EP. 根据蒙特卡洛 模拟分析, 立体选择性主要源于分析物与 MOF 骨架中 特定氨基酸的非共价作用(图 9d), 具体来说，(一)-EP 仅 可与骨架中甘氨酸形成弱的氢键作用, (+)-EP 可与骨架 中组氨酸形成强的氢键作用，还与甘氨酸中氨基和䍨基 都有一定作用，由此实现(十)-EP 的优先吸附(图 9e).

表 1 氨基酸功能化晶态多孔聚合物的不同构筑方法以及应用的对比

Table 1 Synthetic methods and applications of amino acid functionalized crystalline porous polymers

\begin{tabular}{|c|c|c|c|c|}
\hline 氨基酸功能化的晶态多孔聚合物 & 氨基酸 & 构筑方法 & 应用 & 文献 \\
\hline MIL-101-L-Pro & $L$-脯氨酸 & 共价修饰 & $\begin{array}{l}\text { 醇类、胺类、腈类、酯类、醛类等外消旋体 } \\
\text { 的手性拆分 }\end{array}$ & {$[44]$} \\
\hline $\mathrm{Cu}(\mathrm{GHG})$ & 三肽(Gly-L-His-Gly) & 原位构筑 & 脱氧麻黄碱、麻黄碱等手性药物的手性拆分 & {$[45]$} \\
\hline $\mathrm{Ni}_{2}(L-\mathrm{Asp})_{2}$ (bipy) & $L$-天冬氨酸 & 原位构筑 & 2-甲基-2,4-戊二醇外消旋体的手性拆分 & {$[46]$} \\
\hline L-His-ZIF-8/AAO & $L$-组氨酸 & 原位构筑 & 1-苯基乙醇外消旋体的膜手性拆分 & [47] \\
\hline $\begin{array}{l}\text { MIL-53-NH- } L \text {-Glu-based MMMs, } \\
\text { MIL-53-NH- } L \text {-His-based MMMs }\end{array}$ & $(L-$-组氨酸、 $L$-谷氨酸) & 共价修饰 & 1-苯基乙醇外消旋体的膜手性拆分 & {$[48]$} \\
\hline $\mathrm{Zn}_{4}(L-\mathrm{Ser})_{2}(\mathrm{bdc})_{3} \cdot 6 \mathrm{DMF}$ & $L$-丝氨酸 & 原位构筑 & $\mathrm{CO}_{2}$ 的存储以及 $\mathrm{CO}_{2} / \mathrm{N}_{2}$ 吸附分离 & {$[21]$} \\
\hline$\left.\left.[\mathrm{Cd}(\mathrm{L} 2)(\mathrm{Cl})]\left(\mathrm{H}_{2} \mathrm{O}\right)\right] 、[\mathrm{Cd}(\mathrm{L} 3)(\mathrm{Cl})]\left(\mathrm{H}_{2} \mathrm{O}\right)\right]$ & $L$-丝氨酸、 $L$-苏氨酸 & 原位构筑 & 水吸附 & {$[17]$} \\
\hline $\mathrm{Ca}^{\mathrm{II}} \mathrm{Cu}_{6}{ }_{6}^{\mathrm{II}}[(S, S)-\text { methox }]_{3}(\mathrm{OH})_{2}\left(\mathrm{H}_{2} \mathrm{O}\right) \cdot 16 \mathrm{H}_{2} \mathrm{O}$ & $L$-甲硫氨酸 & 原位构筑 & $\mathrm{AuCl}_{3} 、 \mathrm{AuCl}$ 的吸附富集 & {$[49]$} \\
\hline $\mathrm{Ca}^{\mathrm{II}} \mathrm{Cu}_{6}{ }_{6}^{\mathrm{II}}[(S, S) \text {-serimox }]_{3}(\mathrm{OH})_{2}\left(\mathrm{H}_{2} \mathrm{O}\right)$ & $L$-丝氨酸 & 原位构筑 & 维生素 B 吸附 & {$[50]$} \\
\hline ZnGGH & 三肽(Gly-Gly-L-His) & 原位构筑 & $\begin{array}{l}\text { 二氧六环、环戊烷、 } N, N-\text { 二甲基甲酰胺、 } \\
\text { 呋南以及二甲基亚砜等小分子的吸附 }\end{array}$ & {$[25]$} \\
\hline UiO-66-F、UiO-66-W & $L$-苯丙氨酸、 $L$-色氨酸 & 原位调控 & ACE 抑制肽的吸附富集 & {$[34]$} \\
\hline MCNC@COF@GSH & 谷胱甘肽 & 共价修饰 & $N$-连接糖肽的高效富集 & [39] \\
\hline Al-MIL-101-NH2-Gly-Pro & 二肽(L-Gly-Pro) & 共价修饰 & 硝基苯甲醛和丙酮的不对称 Aldol 催化 & [29] \\
\hline CuTrp & $L$-色氨酸 & 原位构筑 & $\mathrm{CO}_{2}$ 与环氧化物的环加成催化 & {$[52]$} \\
\hline MOF-808-his-Cu & $L$-组氨酸 & 共价修饰 & 甲烷高选择性氧化成甲醇催化 & {$[31]$} \\
\hline $\begin{array}{l}\text { MTV- }\left(\mathrm{CH}_{3}\right)_{0.6}\left(\mathrm{CH}_{2} \mathrm{NH}-\text { Ala-Gly-Pro-NH}\right. \\
\text { MTV- }\left(\mathrm{CH}_{3}\right)_{0.1}\left(\mathrm{CH}_{2} \mathrm{NH}-\text { Asp-His-Cys- } \mathrm{NH}_{2}\right)_{0.1}\end{array}$ & $\begin{array}{l}\text { 三肽 } \\
\left(\mathrm{H}_{2} \mathrm{~N}-\mathrm{Pro}-\mathrm{Gly}-\mathrm{Ala}-\mathrm{CONHL} \text {, }\right. \\
\left.\mathrm{H}_{2} \mathrm{~N}-\mathrm{Cys}-\mathrm{His}-\mathrm{Asp}-\mathrm{CONHL}\right)\end{array}$ & 共价修饰 & $\begin{array}{l}\text { 肽链序列(五肽 } \mathrm{H}_{2} \mathrm{~N}-\mathrm{Ala}-\mathrm{Tyr}- \\
\text { Ala-Ser-Ala-CONH} \mathrm{N}_{2} \text { ) 的切割催化 }\end{array}$ & {$[30]$} \\
\hline MIP-202(Zr) & $L$-天冬氨酸 & 原位构筑 & 质子传导 & {$[16]$} \\
\hline
\end{tabular}




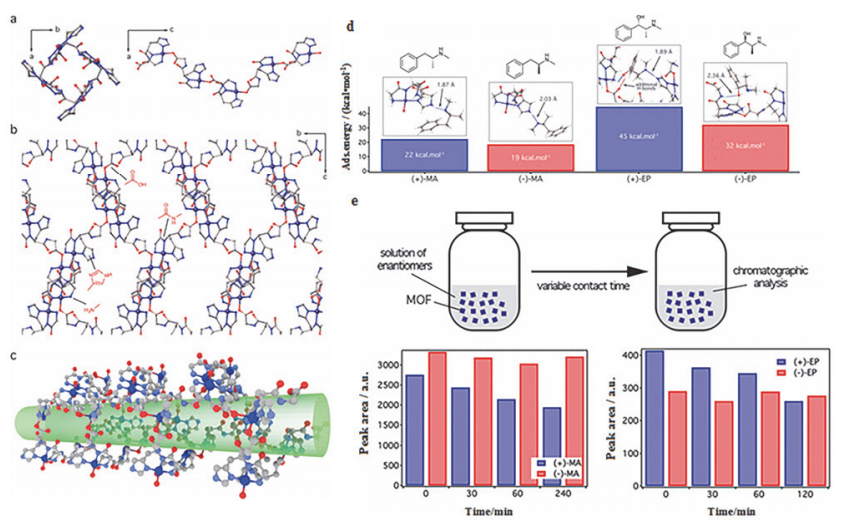

图 9 (a) $\mathrm{Cu}(\mathrm{GHG})$ 中的螺旋链结构. (b) 多肽中官能团修饰的孔道表 面. (c) $\mathrm{Cu}(\mathrm{GHG})$ 中一维孔道的功能位点易形成超分子作用, 适于手性 识别和篮分. (d) $\mathrm{Cu}(\mathrm{GHG})$ 结构中 $(+,-) \mathrm{MA}$ 和 EP 对映体的蒙特卡洛 模拟结合几何构型以及相对于气相计算出的相应吸附能绝对值. (e) $\mathrm{Cu}(\mathrm{GHG})$ 对映选择性识别手性药物 MA 和 EP. 改编自文献[45]

Figure 9 (a) Structure of helicoidal chains in $\mathrm{Cu}(\mathrm{GHG}) \mathrm{MOF}$. (b) Functional groups in the peptidic backbone decorating the surface of the pores. (c) $1 \mathrm{D}$ channels in $\mathrm{Cu}(\mathrm{GHG})$ are surrounded by functional sites prone to establish supramolecular interactions, well suited for chiral recognition and discrimination. (d) Representative MC binding geometries of $(+$, -) MA and EP enantiomers within the structure of $\mathrm{Cu}(\mathrm{GHG})$ and corresponding adsorption energies as absolute values calculated with respect to gas phase. (e) Evolution of the enantioselective recognition of $\mathrm{Cu}(\mathrm{GHG})$ for chiral drugs MA and EP. Adapted from the literature [45]

膜手性拆分技术以其能耗低、操作简单、可批量处 理、可连续操作等优点, 在手性拆分领域得到了广泛的 关注，具有良好的应用前景 ${ }^{[4 a]}$. 金万勤课题组 ${ }^{[46]}$ 报道了 利用 $L$-天冬氨酸和联吡啶(bipy)作为配体原位合成三维 手性 MOF 材料一 $\mathrm{Ni}_{2}(L-\mathrm{Asp})_{2}$ (bipy), 利用高能球磨法获 得了亚微米级大小的 MOF 晶种, 通过浸渍、涂覆将其 涂在陶瓷基底上, 然后通过二次生长制备薄膜. 作者将 其用于外消旋体 2-甲基-2,4-戊二醇的手性识别和吸附 分离实验, 手性拆分 $e e$ 值为 $35.5 \%$. 王焕庭课题组 ${ }^{[47]}$ 报 道了 $L$-组氨酸修饰的 MOF 膜一 $L$-His-ZIF- $8 / \mathrm{AAO}$, 其手 性拆分外消旋 1-苯基乙醇的 $e e$ 值为 $76 \%$. 由于手性 MOFs 在多孔基底上直接生长成膜时通常会形成一定的 缺陷, 导致分离效果不佳. 混合基质膜(MMMs)结合了 MOFs 和聚合物的潜在优点, 可以一定程度上解决薄膜 制备中的缺陷问题. 该课题组 ${ }^{[48]}$ 又合成了两例具有优 异手性拆分性能的手性 MOF 聚合物混合基质膜材料一 MIL-53-NH- $L$-Glu-based MMMs 和 MIL-53-NH- $L$-Hisbased MMMs(图 10a 10f). 作者先将氨基酸 ( $L$-His 或 $L$-Glu) 通过共价修饰引入到 MIL-53- $\mathrm{NH}_{2}$ 骨架上赋予其 手性特征, 再将 MIL-53-NH- $L$-His、MIL-53-NH- $L$-Glu 纳米晶与聚醚砜 PES 混合, 借助溶液浇铸、溶剂蒸发方 法制备自支撑膜, 并通过对混合基质膜的 MOF 负载量 的调控, 实现薄膜对外消旋体 1-苯基乙醇的高效手性拆 分效果, ee 值高达 $100 \%$.

\section{2 吸附}

晶态多孔聚合物材料具有高比表面积、发达的孔道
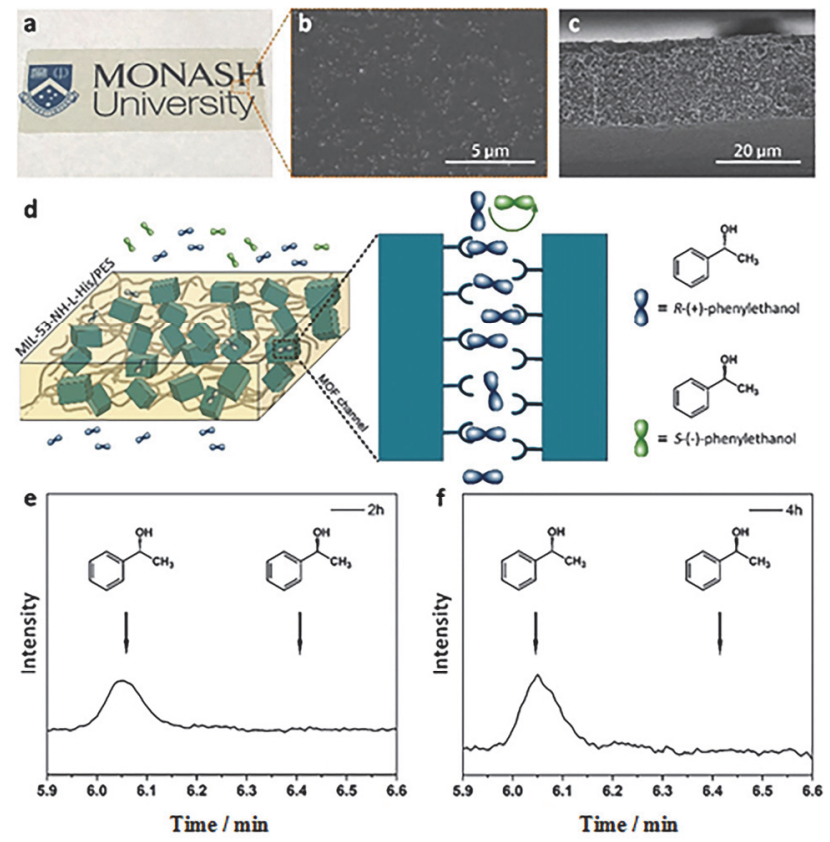

图 10 手性分离 1-苯乙醇对映体的 MOF 基 MMMs 的制备与表征. 改 编自文献[48]

Figure 10 Fabrication and characterization of homochiral MOF-based MMMs for chiral separation of 1-phenylethanol enantiomers. Adapted from the literature [48]

结构、丰富的孔道环境，可以有效容纳特定的客体分子， 表现出高吸附容量和选择性, 在化工、医药、环境等领 域具有重要的应用. 氨基酸功能化晶态多孔聚合物可以 从孔表面的性质、孔道尺寸和形状、骨架的柔性化等方 面调控主客体作用，进而调控其在气、液相的吸附性能.

MOF 材料的孔道表面修饰极性氨基酸基团，可以 提高其对 $\mathrm{CO}_{2}$ 的亲和力, 进而提高吸附容量和选择性. 张健课题组 ${ }^{[21]}$ 合成了基于丝氨酸的三维微孔手性 MOF 材料 $-\mathrm{Zn}_{4}(L-\mathrm{Ser})_{2}(\mathrm{bdc})_{3} \cdot 6 \mathrm{DMF}(1-\mathrm{L})$ 和 $\mathrm{Zn}_{4}(D-\mathrm{Ser})_{2}-$ $(\mathrm{bdc})_{3} \cdot 6 \mathrm{DMF}(1-\mathrm{D})$, 这两种材料具有相似的结构, 考虑 到 1-L 材料更容易移除孔道中的 DMF, 以 1-L 展开吸附 实验. 结果显示经过高温真空处理的 1- $\mathrm{L}$ 材料对 $\mathrm{CO}_{2}$ 的 吸附选择性和吸附容量优于 $\mathrm{N}_{2}$ 和 CO. 在 $195 \mathrm{~K}$ 和 0.1 $\mathrm{MPa}$ 下, 1-L 对 $\mathrm{CO}_{2}$ 吸附量为 $127 \mathrm{~cm}^{3} / \mathrm{g}$; 在 $273 \mathrm{~K}$ 和 0.1 $\mathrm{MPa}$ 下, 其 $\mathrm{CO}_{2} / \mathrm{N}_{2}$ 吸附选择性为 $185: 1$. 氨基酸还可 以调节 MOF 材料孔道的亲水性环境, 提高水吸附效果. Banerjee 等 ${ }^{[17]}$ 报道了一系列基于氨基酸衍生物的 MOF, 以 $\left.[\mathrm{Cd}(\mathrm{L} 2)(\mathrm{Cl})]\left(\mathrm{H}_{2} \mathrm{O}\right)\right] \infty(\mathbf{6 a})$ 和 $\left[\mathrm{Cd}(\mathrm{L} 3)(\mathrm{Cl})\left(\mathrm{H}_{2} \mathrm{O}\right)_{2}\right] \infty(7 \mathbf{a})$ 为 例, 其中 L2、 L3 分别是吡啶功能化的 $L$-丝氨酸、 $L$-苏 氨酸. 水吸附测试显示吸附等温线存在滞回环, $6 \mathrm{a}$ 和 7a 分别具有 $120 、 140 \mathrm{~cm}^{3} \cdot \mathrm{g}^{-1}$ (STP) 的吸附量, 证实了孔道 表面与水分子之间存在一定的相互作用，其中 $\mathbf{7 a}$ 结构 中层间的高度亲水性使得水分子和骨架之间形成强的 氢键作用。

氨基酸修饰的功能化孔道还可提高客体分子的特 
异性吸附能力. 例如, Pardo 等 ${ }^{[49]}$ 报道了基于高度柔性的 甲硫氨酸的 $\mathrm{MOF}$ 材料 $-\mathrm{Ca}^{\mathrm{II}} \mathrm{Cu}_{6}{ }_{6}[(S, S) \text {-methox }]_{3}$ $(\mathrm{OH})_{2}\left(\mathrm{H}_{2} \mathrm{O}\right) \cdot 16 \mathrm{H}_{2} \mathrm{O}$, 单晶结构分析表明该材料具有三维 的框架结构, 其中甲硫氨酸的硫醚基团占据在框架的孔 道内部. 作者将其用于金离子的吸附实验，结果显示该 $\mathrm{MOF}$ 对 $\mathrm{AuCl}_{3} 、 \mathrm{AuCl}$ 的吸附量分别为 598 和 $300 \mathrm{mg} / \mathrm{g}$, 并且在含有其他金属阳离子如 $\mathrm{Pd}^{2+} 、 \mathrm{Ni}^{2+} 、 \mathrm{Cu}^{2+} 、 \mathrm{Zn}^{2+}$ 和 $\mathrm{Al}^{3}$ 等情况下, 也表现出特异性可逆吸附行为. Herrero-Martinez 等 ${ }^{[50]}$ 合成了 $L$-丝氨酸衍生物功能化的 草酰胺配体(serimox), 将其与 $\mathrm{Ca}^{2+} 、 \mathrm{Cu}^{2+}$ 反应制备了双 金属 MOF 材料 - $\mathrm{Ca}^{\mathrm{II}} \mathrm{Cu}_{6}{ }_{6}[(S, S) \text {-serimox }]_{3}(\mathrm{OH})_{2}\left(\mathrm{H}_{2} \mathrm{O}\right)$, 单晶结构显示其孔径的大小、形状与客体分子匹配, 并 且亲水性的六边形通道中限域了大量 $L$-丝氨酸的羟基 官能团, 可以分别与四种亲水性的维生素 B 发生超分子 主客体作用, 从而对果汁和能量饮料中的维生素 $\mathrm{B}$ 进行 有效的分子识别和萃取吸附作用. 与商业的 C18 固相萃 取柱相比, 该 MOF 材料具有用量小、可再生能力强、 分析物损失小等优点.

柔性多肽单元的引入, 使得具有动态特征的柔性 MOFs 在吸附方面表现出特异性响应行为. Rosseinsky 等 $^{[25]}$ 利用三肽作为配体制备了三维柔性 $\mathrm{MOF}$ 材料 $-\mathrm{ZnGGH}$ (图 11a 11c), 基于短肽连接单元中共价键的 旋转, 这种 MOF 材料可以响应化学环境的变化诱导短 肽的构象发生改变, 在九个不同的晶体结构中灵活变

a
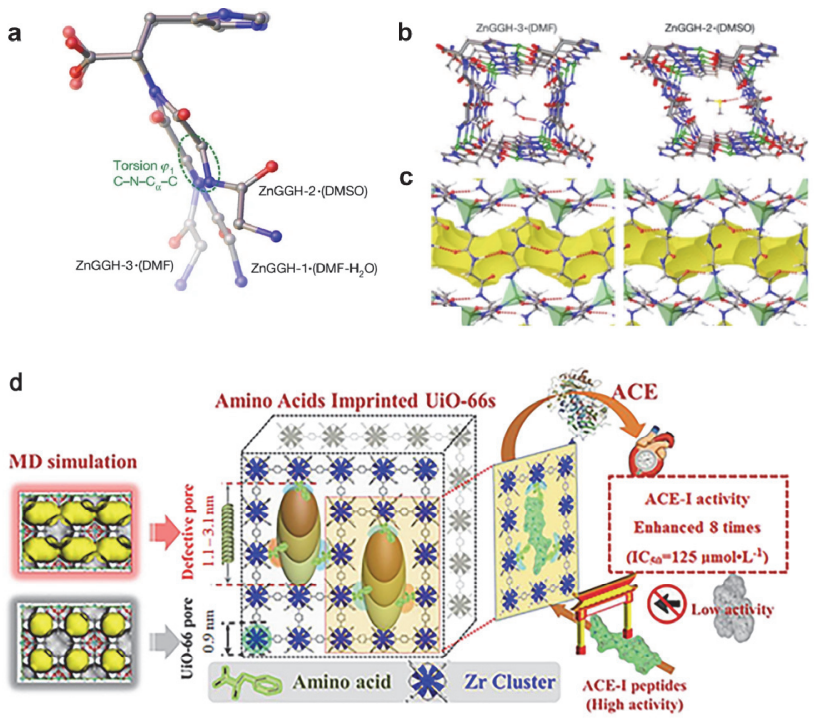

图 11 (a) 三肽 $\mathrm{GGH}$ 配体在 $\mathrm{ZnGGH}-1 \cdot\left(\mathrm{DMF}-\mathrm{H}_{2} \mathrm{O}\right) 、 \mathrm{ZnGGH}-2$ • (DMSO) 和 ZnGGH-3•(DMF) 在精修晶体结构中的构象. (b) ZnGGH-3 • (DMF)和 $\mathrm{ZnGGH}-2 \cdot(\mathrm{DMSO})$ 中一维孔道俯视图. (c) 侧视图揭示了每 种结构中的骨架内氢键模式. (d) 氨基酸印迹 UiO-66s 可高度识别吸 附血管紧张素转换酶抑制肽过程. 改编自文献[25], [34]

Figure 11 (a) Conformation of the GGH linker in the refined experimental crystal structures $\mathrm{ZnGGH}-1 \cdot\left(\mathrm{DMF}-\mathrm{H}_{2} \mathrm{O}\right), \mathrm{ZnGGH}-2 \cdot(\mathrm{DMSO})$ and $\mathrm{ZnGGH}-3 \cdot(\mathrm{DMF})$. (b) The view down the one-dimensional pores of ZnGGH-3•(DMF) and ZnGGH-2•(DMSO). (c) The side view reveals the intraframework hydrogen-bond pattern for each structure. (d) Amino acid imprinted UiO-66s for highly recognized adsorption of small angiotensinconverting-enzyme-inhibitory peptides. Adapted from the literature [25], [34]
换，从而触发 MOFs 对二氧六环、环戊烷、 $N, N$-二甲基 甲酰胺(DMF)、呋喃以及二甲基亚砜(DMSO)等小分子 客体的吸附摄取.

框架材料中氨基酸、肽段的引入，可促进多肽在孔 道中的选择性吸附和富集. 前文中已提到赵钟兴课题 组 $^{[34]}$ 合成了 $L$ - 苯丙氨酸、 $L$-色氨酸印迹的缺陷型 MOFs-UiO-66-F 和 UiO-66-W(图 11d), 氨基酸不仅可 以调控 MOF 的缺陷, 而且可在缺陷位点处进行修饰. 将其用于血管紧张素转换酶(ACE)抑制肽的吸附, 结果 显示 UiO-66-F 和 UiO-66-W 对 DDFF、DDWW (D 为天 冬氨酸、 $F$ 为苯丙氨酸、 $W$ 为色氨酸)两种四肽的吸附 量分别为 1.25 和 $1.37 \mathrm{mmol} \cdot \mathrm{g}^{-1}$, 选择性分别达到 249 和 279. 与原始的 UiO-66 相比, 氨基酸印迹的 MOF 材 料对于 ACE 抑制肽的富集容量和选择性都有显著提升. 蓝芳课题组 ${ }^{[39]}$ 报道了一例谷胱甘肽功能化的磁性 COF 核壳结构微球-MCNC@COF@GSH, 由于尺寸篮分效 应和亲水的孔道环境, $\mathrm{COF}$ 微球可以高效富集 $\mathrm{N}$-连接糖 肽, 表现出高选择性、高灵敏度、可循环使用性, 因而 有望应用于复杂生物样品的分析.

\section{3 催化}

由于具有发达的孔道结构, 以及骨架中均匀分布的 金属中心和各类官能团, 晶态多孔聚合物在催化领域展 现出巨大的应用潜力. 氨基酸和短肽可作为活性有机催 化功能基团, 将其引入晶态多孔聚合物体系, 不仅可以 提供催化位点，而且可以对框架中金属中心配位空间的 组成、催化活性位点、空腔进行合理设计和修饰, 进而 提高催化反应中底物的转化率、立体或手性选择性.

通过氨基酸、多肽功能化，可在 MOFs 中引入手性 催化位点, 制备高性能的手性催化剂. Van Der Voort 等 ${ }^{[51]}$ 采用 $L$-脯氨酸作为 Zr-MOFs 生长过程的调节剂, 成功在骨架中引入手性催化位点. 以氨基酸功能化的 UiO-66-LP 作为手性催化剂, 在硝基苯甲醛和丙酮的不 对称 Aldol 反应中表现出较高的催化活性和非对映立体 选择性; 循环使用三次后, 非对映选择性保持不变, 转 化率略有下降. Farrusseng 等 ${ }^{[29]}$ 借助 MOF 骨架中的氨基 与氨基酸或多肽的羧基发生脱水缩合反应制备了一系 列手性多肽官能化 MOFs，其中 Al-MIL-101- $\mathrm{NH}_{2}-$ Gly-Pro 框架中含有 L-Pro 活性基团, 作者将其用于催化 硝基苯甲醛和丙酮的不对称 Aldol 反应，实现了 $95 \%$ 以 上的产率和 $17 \%$ 的对映选择性.

MOF 材料中的金属中心也可作为催化位点, 氨基 酸与金属中心的配位方式对 MOFs 催化活性有着明显的 影响. Won Park 等 ${ }^{[52]}$ 以 $L$-色氨酸作为配体合成了铜基 MOF 材料 CuTrp, 在无溶剂条件下 CuTrp 与助催化剂四 丁基溴化铵用于协同催化 $\mathrm{CO}_{2}$ 与环氧化物的环加成反 应，其中环氧氯丙烷的反应达到 $98.6 \%$ 的转化率和 $99.1 \%$ 的选择性. 实验结果和密度泛函理论显示, 其反 应机理与 CuTrp 结构中五配位铜中心(活性路易斯酸性 
位点), 以及助催化剂亲核溴化物阴离子协助环氧化物 的开环有关联. 该催化剂还表现出良好的水热稳定性, 且加入少量水可以提升它的催化活性. 此外, 即使在三 次循环实验后仍能保持其催化活性, 铜离子的浸出率仅 为 $0.08 \%$.

MOF 材料高度有序的多孔结构可以作为“支架”, 通过修饰氨基酸、短肽等构筑仿酶的催化活性位点, 利 用不同骨架结构调控其二级、三级结构, 实现高效催化 过程. 受颗粒性甲烷单加氧酶( $\mathrm{pMMO}$ )结构的启发, Yaghi 课题组 ${ }^{[31]}$ 在 MOF-808 上修饰组氨酸引入咪唑单元, 经过金属化构筑 $\mathrm{Cu}-\mathrm{O}$ 复合物作为催化活性位点(图 12a), 其微孔结构可作为底物扩散通道. 在 $150{ }^{\circ} \mathrm{C}$ 等温条件 下, MOF 催化剂可以实现甲烷高选择性氧化成甲醇, 密 度泛函理论计算表明双核铜-氧结构可能是其催化位点. 此外, 该课题组 ${ }^{[30]}$ 还利用七步连续的合成后修饰反应 在 MOF 骨架上引入特定氨基酸序列, 模拟酶的活性位 点的空间排布和组分异质性, 在 MOF 孔道中构筑类酶 的复杂环境实现高选择性催化. 氨基酸序列功能化 MOF 材料 MTV- $\left(\mathrm{CH}_{3}\right)_{0.6}\left(\mathrm{CH}_{2} \mathrm{NH}-\mathrm{Ala}-\mathrm{Gly}-\mathrm{Pro}-\mathrm{NH}_{2}\right)_{0.1}$ 作 为催化剂, 在丁醛的 $\alpha$-氯化反应中表现出较高的立体选 a

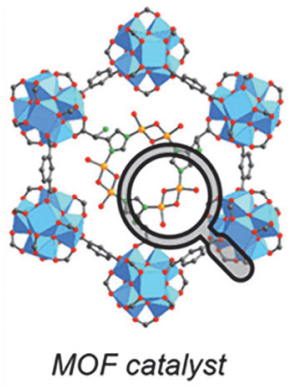

b

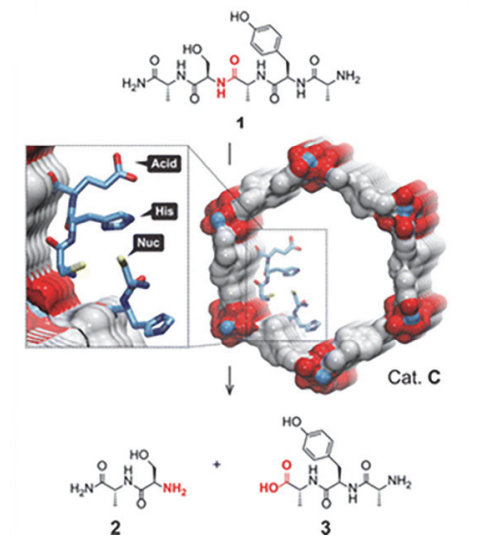

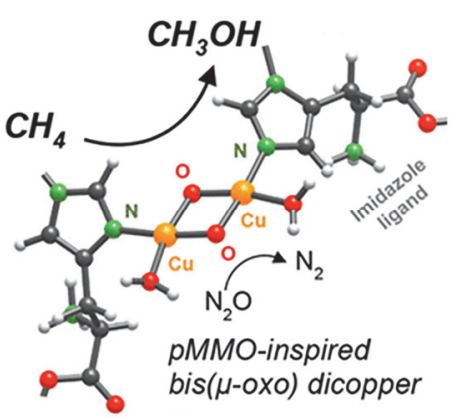

bis $(\mu$-oxo) dicopper
图 12 (a) 仿生 MOF 催化剂选择性氧化甲烷到甲醇; (b) 催化剂 [MTVIRMOF-74-III- $\left(\mathrm{CH}_{3}\right)_{0.6}\left(\mathrm{CH}_{2} \mathrm{NH}-\mathrm{Asp}-\mathrm{His}-\mathrm{Cys}-\mathrm{NH}_{2}\right)_{0.1}$ ] 催化切割五 肽, 以及类烟草蚀纹病毒(TEV)内肽酶及其结构中参与催化的三种氨 基酸. 改编自文献[30], [31]

Figure 12 (a) Bioinspired metal-organic framework catalysts for selective methane oxidation to methanol. (b) Catalytic cleavage of pentapeptide 1 by Cat. C [MTVIRMOF-74-III- $\left(\mathrm{CH}_{3}\right)_{0.6}\left(\mathrm{CH}_{2} \mathrm{NH}-\mathrm{Asp}\right.$-His-Cys$\left.\left.\mathrm{NH}_{2}\right)_{0.1}\right]$ in the specific sequence containing serine, and cartoon representation of the enzyme TEV endoprotease, highlighting the three amino acids that participate in the catalysis. Adapted from the literature [30], [31]
择性. 相较于脯氨酸均相催化反应, 这种非对称性诱导 作用的提升可能是由于 MOF 孔道内立体化学限制作用. 此外，作者利用类烟草蚀纹病毒(TEV)内肽酶的氨基酸 序列修饰 MOF 框架, MTV- $\left(\mathrm{CH}_{3}\right)_{0.6}\left(\mathrm{CH}_{2} \mathrm{NH}-\mathrm{Asp}-\mathrm{His}-\right.$ Cys- $\left.\mathrm{NH}_{2}\right)_{0.1}$ 成功实现特定肽链序列(五肽 $\mathrm{H}_{2} \mathrm{~N}-\mathrm{Ala}-\mathrm{Tyr}-$ Ala-Ser-Ala-CONH${ }_{2}$ )的切割(图 12b), 初步证明了 $\mathrm{MOF}$ 骨架上多肽序列的调控可以从复杂性和空间上实现类 酶催化转化行为.

\section{4 质子传导}

质子交换膜是质子交换膜燃料电池(PEMFC) 的核 心组成之一，其离子传导能力直接影响着然料电池的性 能. 由于具有高比表面积、丰富的结构、可调控的孔道 环境, MOFs 在质子传导方面具有一定的优势. 通过氨 基酸功能化, 在 MOFs 骨架上引入酸性位点如羧基、羟 基等，提高质子载流子浓度和迁移率; 此外, 孔道中的 $\mathrm{NH}_{3}{ }^{+}$等可以与水形成氢键网络作为质子迁移通道, 提 高质子迁移率.

Serre 等 ${ }^{[16]}$ 报道的基于天冬氨酸的锆基 MOF 材料 MIP-202(Zr)(图 13a 13f), 在温度为 $363 \mathrm{~K}$ 、相对湿度 为 $95 \%$ 的条件下，其质子电导率可达到 $0.011 \mathrm{~S} \cdot \mathrm{cm}^{-1}$; 且材料在较宽 $\mathrm{pH}$ 范围的溶液和沸水中, 都表现出良好 的化学稳定性. 作者结合实验测试和蒙特卡洛模拟分析 来研究其质子传导机理, 发现吸附在其孔道中的水分子 可以通过氢键作用形成水分子簇，且可与孔壁上丰富的 质子源 $\mathrm{NH}_{3}{ }^{+}$基团进一步形成更加拓展的氢键网络, 为 质子传导提供路径. MIP-202(Zr)不仅具有优异的质子传 导能力, 而且制备方法简单、绿色、可大批量，原料成 本低，因而具有实际应用的潜力.
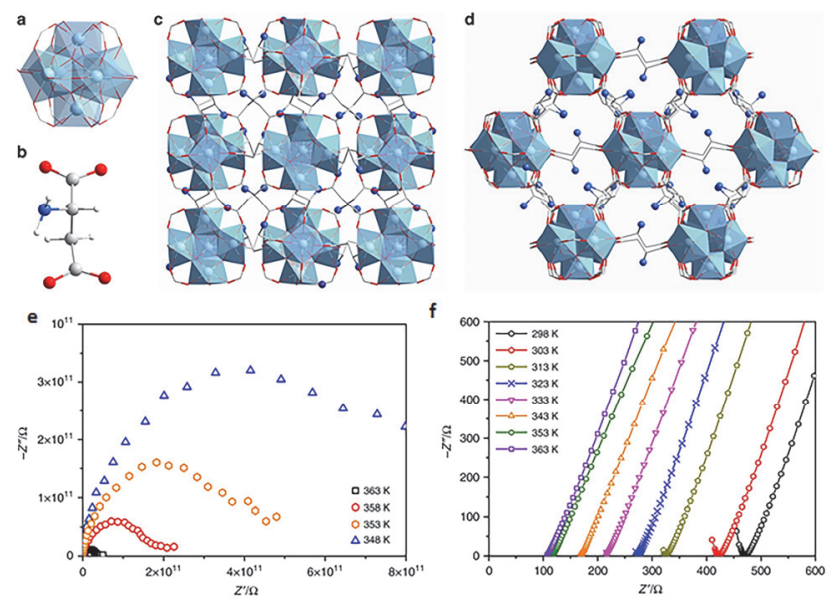

图 13 (a) 12 连接的 $\mathrm{Zr}_{6}\left(\mu_{3}-\mathrm{O}\right)_{4}\left(\mu_{3}-\mathrm{OH}\right)_{4}(\mathrm{COO}-)_{12}$ 次级结构单元. (b) 天 冬氨酸配体. (c) MIP-202( $\mathrm{Zr}$ ) 晶体结构沿 $a$ 轴方向的视图. (d) MIP-202(Zr)晶体结构沿(101)晶面的视图. (e)和(f) MIP-202(Zr)在相对 湿度为 $0 \%$ 和 $95 \%$ 时的交流阻抗数据的 Nyquist 图. 改编自文献[16] Figure 13 (a) The 12-connected $\mathrm{Zr}_{6}\left(\mu_{3}-\mathrm{O}\right)_{4}\left(\mu_{3}-\mathrm{OH}\right)_{4}(\mathrm{COO}-)_{12}$ cluster SBU. (b) An aspartic acid linker. (c) The crystal structure of MIP-202(Zr) viewed along the $a$-axis. (d) The crystal structure of MIP-202(Zr) viewed along the (101) plane. (e) and (f) Nyquist plots from AC impedance data for MIP-202(Zr) at $0 \%$ and $95 \%$ RH. Adapted from the literature [16] 


\section{5 总结与展望}

近年来, 氨基酸功能化晶态多孔聚合物材料的设 计、制备、应用等方面取得了较大的发展. 借助氨基酸 丰富的官能团、配位方式、物理化学性质, 在 MOFs、 $\mathrm{COFs}$ 的构筑过程中, 氨基酸可以直接作为构筑单元或 是通过合成后修饰的方式连接到骨架结构中, 赋予框架 材料丰富的结构、特殊的孔道环境(手性、极性、亲疏 水性等)、活性催化位点、特异性吸附位点等; 或是作为 调节剂调控成键过程和晶体生长过程. 这些特征进一步 拓展了 $\mathrm{MOF}$ 和 $\mathrm{COF}$ 材料在手性拆分、吸附、催化、质 子传导等方向中的应用.

尽管氨基酸功能化晶态多孔聚合物展现出特殊的 功能特点和广阔的应用前景, 但是目前相关研究还处于 初始阶段, 仍面临着许多困难与挑战. 首先, 由于氨基 酸结构的相对柔性和低对称性, 以氨基酸或其衍生物作 为构筑单元合成框架材料具有较大的难度, 并且很难对 骨架结构进行预判或调控, 尤其是合成基于氨基酸单元 的 COF 材料更具挑战性, 因此开发新的合成方法和策 略, 对于丰富氨基酸功能化 COFs 和 MOFs 的种类和结 构十分重要. 其次, 通过合成后修饰引入氨基酸过程中, 常存在修饰程度难以控制的问题. 此外, 对于氨基酸在 孔道中的空间排布和构型构象难以精确调控, 限制了在 吸附和催化等应用中构效关系的研究. 再次, 提升氨基 酸功能化晶态多孔聚合物的化学稳定性和热稳定性是 拓展其应用领域的重要前提之一, 高稳定性材料的开发 和修饰策略亟待发展和完善.

目前, 氨基酸功能化晶态多孔聚合物的研究主要集 中在 MOF 材料领域, 而面向 COF 材料的研究相对较少. COF 材料孔道尺寸相对较大 $(0.64 \sim 5.8 \mathrm{~nm})$ 、热稳定性 优异, 在催化、吸附、分离等应用场景中具有较大的优 势. 然而, 在 COFs 体系中引入氨基酸、肽链等生物活 性组分面临一定的困难: 一方面, 原位引入的策略受到 COFs 相对苛刻的生长条件的限制, 并且柔性构筑单元 可能会影响骨架的稳定性和结晶度; 另一方面, 通过合 成后修饰的方法引入, 对于 COFs 的化学稳定性有较高 的要求. 随着 $\mathrm{COF}$ 材料领域的发展, 其构筑单元的种类 日渐丰富, 合成方法、修饰策略也不断完善和发展, 大 大提高了其结晶度和稳定性, 因此氨基酸功能化的 $\mathrm{COF}$ 材料极具发展前景.

氨基酸作为构成蛋白质的基本单元, 具有丰富的生 物学功能, 在酶催化过程中也发挥着重要作用; 将氨基 酸引入 MOFs、COFs 体系中, 结合其有序的结构、开放 的骨架、发达的孔道等特点, 在生物医药、催化等领域 中极具应用前景, 同时也面临着挑战. 氨基酸单元或序 列等生物活性分子作为配体构筑晶态多孔聚合物, 可在 一定程度上提高框架材料的生物相容性和降解性, 因此 有望作为医药载体或成像剂载体, 用于药物缓释、生物 成像，但其实际应用还受到多方面的制约：首先，氨基
酸单元或序列引入框架材料, 可能会导致其活性及功能 的部分损失，如何最大程度的保证其活性十分重要; 其 次, 作为药物传递载体, 要求其在传递过程中保持良好 的稳定性、避免药物的提前释放, 而在到达目标位点时 实现药物的可控释放，同时保证材料可以分解被人体代 谢排出体外，因此，材料在生物体系的稳定性和生物可 降解性之间的调控也亟需探究; 最后, 对于氨基酸衍生 物、混合配体构筑的体系，材料长期的毒副作用仍待进 一步考察. 总的来说, 材料的活性、稳定性、生物可降 解性以及生物安全性都是拓展其生物应用的关键. 在催 化应用中, 利用氨基酸的官能团, 在框架材料中构筑仿 酶催化活性位点, 有望实现温和条件下高效的 “类酶” 催化行为, 其中氨基酸的修饰程度、空间排布、构型构 象以及位点周围的微环境的调控是研究中的关键科学 问题.

在本综述中, 主要介绍了氨基酸功能化 $\mathrm{MOF}$ 和 COF 材料的制备策略，及其在手性拆分、吸附、催化、 质子传导领域的应用. 在此基础上，我们总结了目前该 领域存在的挑战以及未来的发展方向, 为氨基酸功能化 晶态多孔聚合物的设计与发展提供了研究思路. 我们相 信，以这类材料为代表的生物活性分子功能化多孔晶态 材料是未来生物医药材料、仿生材料等领域的研究热 点.

\section{作者简介}

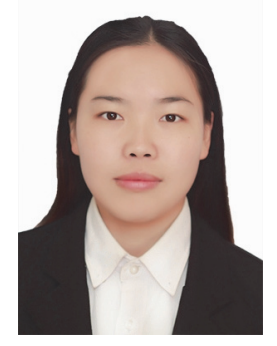

梅佩, 北京理工大学化学与化工学院, 2018 级在读硕士研 究生(化学工程专业), 本科毕业于黄冈师范学院(应用化学专 业), 现主要从事氨基酸功能化晶态多孔材料的合成与应用研 究.

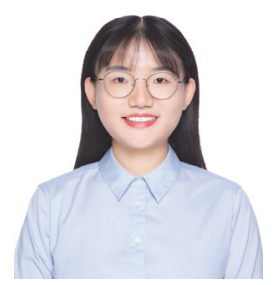

张媛媛, 北京理工大学前沿交叉科学研究院, 预聘助理 教授. 2013 年 2019 年在北京理工大学化学与化工学院获得 博士学位, 2017 年 2018 年在美国西北大学化学系访问学习 (联合培养博士), 2019 年就职于北京理工大学前沿交叉科学研 究院．主要从事金属有机框架(MOF)、共价有机框架 $(\mathrm{COF})$ 等 
功能多孔材料的可控制备和柔性加工, 及其在分离、催化、智 能响应等领域的应用研究.

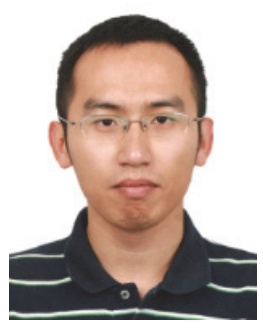

冯霄, 北京理工大学博士生导师, 化学与化工学院教授, 国家自然科学基金优秀青年科学基金获得者. 分别于 2008 年 和 2013 年于北京理工大学材料学院取得本科与博士学位, 攻 读博士期间以联合培养博士研究生身份留学于日本国家自然 科学研究机构——分子科学研究所. 2013 年就职于北京理工 大学化学与化工学院. 主要从事关于共价有机框架材料等晶 态多孔材料的构效关系研究以及膜分离相关领域应用研究.

\section{References}

[1] (a) Ploetz, E.; Engelke, H.; Lächelt, U.; Wuttke, S. Adv. Funct. Mater. 2020, DOI: 10.1002/adfm.201909062. (b) Ferey, G. Chem. Soc. Rev. 2008, 37, 191. (c) Ferey, G.; Mellot-Draznieks, C.; Serre, C.; Millange, F. Acc. Chem. Res. 2005, 38, 217. (d) Horike, S.; Shimomura, S.; Kitagawa, S. Nat. Chem. 2009, 1, 695. (e) Kitagawa, S.; Kitaura, R.; Noro, S. Angew. Chem. Int. Ed. 2004, 43, 2334. (f) Yaghi, O. M. Mol. Front. J. 2019, 03, 66.

[2] (a) Wang, Q.; Astruc, D. Chem. Rev. 2019, 120, 1438. (b) Stock, N.; Biswas, S. Chem. Rev. 2012, 112, 933. (c) Rowsell, J. L. C.; Yaghi, O. M. Micropor. Mesopor. Mat. 2004, 73, 3. (d) Furukawa, H.; Ko, N.; Go, Y. B.; Aratani, N.; Choi, S. B.; Choi, E.; Yazaydin, A. O.; Snurr, R. Q.; O'Keeffe, M.; Kim, J.; Yaghi, O. M. Science 2010, 329, 424.

[3] (a) Lohse, M. S.; Bein, T. Adv. Funct. Mater. 2018, 28, 1705553. (b) Geng, K.; He, T.; Liu, R.; Dalapati, S.; Tan, K. T.; Li, Z.; Tao, S.; Gong, Y.; Jiang, Q.; Jiang, D. Chem. Rev. 2020, DOI: 10.1021/acs.chemrev.9b00550. (c) Huang, N.; Wang, P.; Jiang, D. Nat. Rev. Mater. 2016, 1, 16068.

[4] (a) Zhao, X.; Wang, Y.; Li, D. S.; Bu, X.; Feng, P. Adv. Mater. 2018, 30, 1705189. (b) Zeng, Y.; Zou, R.; Zhao, Y. Adv. Mater. 2016, 28, 2855. (c) Wang, Y.; Huang, N. Y.; Shen, J. Q.; Liao, P. Q.; Chen, X. M.; Zhang, J. P. J. Am. Chem. Soc. 2018, 140, 38. (d) Rodenas, T.; Luz, I.; Prieto, G.; Seoane, B.; Miro, H.; Corma, A.; Kapteijn, F.; Llabrés i Xamena, F. X.; Gascon, J. Nat. Mater. 2014, 14, 48. (e) Furukawa, H.; Cordova, K. E.; O'Keeffe, M.; Yaghi, O. M. Science 2013, 341, 974. (f) Eddaoudi, M.; Kim, J.; Rosi, N.; Vodak, D.; Wachter, J.; O'Keeffe, M.; Yaghi, O. M. Science 2002, 295, 469. (g) Guo, Z.; Zhang, Y.; Feng, X. Acta Chim. Sinica 2020, 78, 397 (in Chinese). (郭振涁, 张媛媛, 冯霄, 化学学报, 2020, 78, 397.) (h) Peng, Z.; Ding, H.; Chen, R.; Gao, C.; Wang, C. Acta Chim. Sinica 2019, 77, 681. (in Chinese). (彭正康, 丁慧敏, 陈如凡, 高超, 汪 成, 化学学报, 2019, 77, 681.)

[5] (a) Pascanu, V.; Gonzalez Miera, G.; Inge, A. K.; Martin-Matute, B. J. Am. Chem. Soc. 2019, 141, 7223. (b) Liu, Y.; Xuan, W.; Cui, Y. Adv. Mater. 2010, 22, 4112. (c) Kang, Y.-S.; Lu, Y.; Chen, K.; Zhao, Y.; Wang, P.; Sun, W.-Y. Coordin. Chem. Rev. 2019, 378, 262. (d) Ding, S. Y.; Gao, J.; Wang, Q.; Zhang, Y.; Song, W. G.; Su, C. Y.; Wang, W. J. Am. Chem. Soc. 2011, 133, 19816. (e) Liu, J.; Zhang, M.; Wang, N.; Wang, C.; Ma, L. Acta Chim. Sinica 2020, 78, 311 (in Chinese). (刘建国, 张明月, 王楠, 王晨光, 马隆龙, 化学学报, 2020, 78, 311.) (h) Hang, G.; Chen, Z.; Jiang, H. Acta Chim. Sinica 2016, 74, 113. (in Chinese). (黄刚, 陈玉贞, 江海龙, 化学学报, 2016, 74, 113.) (i) Xiao, J.; Li, D.; Jiang, H. Sci. Sin. Chim. 2018, 48, 1058.(in Chinese). (肖娟定, 李丹丹, 江海龙, 化学学报, 2018, 48, 1058.) (j) Cai, G.; Ding, M.; Wu, Q.; Jiang, H. Natl. Sci. Rev. $2020,7,37$.

[6] (a) Wales, D. J.; Grand, J.; Ting, V. P.; Burke, R. D.; Edler, K. J.; Bowen, C. R.; Mintova, S.; Burrows, A. D. Chem. Soc. Rev. 2015, 44, 4290. (b) Hussain, M.; Wackerlig, J.; Lieberzeit, P. A. Biosensors
2013, 3, 89. (c) Kong, B.; Selomulya, C.; Zheng, G. F.; Zhao, D. Y. Chem. Soc. Rev. 2015, 44, 7997.

[7] (a) Horcajada, P.; Serre, C.; Vallet-Regi, M.; Sebban, M.; Taulelle, F.; Ferey, G. Angew. Chem. Int. Ed. 2006, 45, 5974. (b) Horcajada, P.; Gref, R.; Baati, T.; Allan, P. K.; Maurin, G.; Couvreur, P.; Ferey, G.; Morris, R. E.; Serre, C. Chem. Rev. 2012, 112, 1232. (c) Zeng, J.; Wang, X.; Zhang, X.; Zhou, R. Acta Chim. Sinica 2019, 77, 1156. (in Chinese). (曾锦跃, 王小双, 张先正, 卓仁禧, 化学学报, 2019, 77, 1156.)

[8] (a) Mu, J.; He, L.; Huang, P.; Chen, X. Coordin. Chem. Rev. 2019 399, 213039. (b) Cai, H.; Huang, Y.-L.; Li, D. Coordin. Chem. Rev. 2019, 378, 207. (c) Anderson, S. L.; Stylianou, K. C. Coordin. Chem. Rev. 2017, 349, 102.

[9] Broer, S.; Broer, A. Biochem. J. 2017, 474, 1935.

[10] Uneyama, H.; Kobayashi, H.; Tonouchi, N. Adv. Biochem. Eng./Biotechnol. 2017, 159, 273.

[11] Bischoff, R.; Schluter, H. J. Proteomics 2012, 75, 2275.

[12] Anokhina, E. V.; Jacobson, A. J. J. Am. Chem. Soc. 2004, 126, 3044.

[13] Li, J.; Pu, T.; Huang, B.; Hou, X.; Zhao, C.; Xie, L.; Chen, L. J. Colloid Interface Sci. 2018, 531, 360

[14] Altun, Ö.; Bilcen, S. Spectrochim. Acta, Part A 2010, 75, 789.

[15] Kathalikkattil, A. C.; Roshan, R.; Tharun, J.; Babu, R.; Jeong, G. S.; Kim, D. W.; Cho, S. J.; Park, D. W. Chem. Commun. 2016, 52, 280.

[16] Wang, S.; Wahiduzzaman, M.; Davis, L.; Tissot, A.; Shepard, W.; Marrot, J.; Martineau-Corcos, C.; Hamdane, D.; Maurin, G.; Devautour-Vinot, S.; Serre, C. Nat. Commun. 2018, 9, 4937.

[17] Kundu, T.; Sahoo, S. C.; Banerjee, R. Cryst. Growth Des. 2012, 12, 4633.

[18] Boer, S. A.; Turner, D. R. CrystEngComm 2017, 19, 2402.

[19] Chen, C.; Li, H. Z. Anorg. Allg. Chem. 2019, 645, 888.

[20] Wang, S. H.; Zheng, F. K.; Zhang, M. J.; Liu, Z. F.; Chen, J.; Xiao, Y.; Wu, A. Q.; Guo, G. C.; Huang, J. S. Inorg. Chem. 2013, 52, 10096.

[21] Tan, Y.-X.; He, Y.-P.; Zhang, J. Inorg. Chem. 2011, 50, 11527.

[22] Li, M.-Y.; Wang, F.; Gu, Z.-G.; Zhang, J. RSC Adv. 2017, 7, 4872.

[23] Zhao, J.; Li, H.; Han, Y.; Li, R.; Ding, X.; Feng, X.; Wang, B. J. Mater. Chem. A 2015, 3, 12145.

[24] Kutzscher, C.; Hoffmann, H. C.; Krause, S.; Stoeck, U.; Senkovska, I.; Brunner, E.; Kaskel, S. Inorg. Chem. 2014, 54, 1003.

[25] Katsoulidis, A. P.; Antypov, D.; Whitehead, G. F. S.; Carrington, E. J.; Adams, D. J.; Berry, N. G.; Darling, G. R.; Dyer, M. S.; Rosseinsky, M. J. Nature 2019, 565, 213.

[26] (a) Deria, P.; Mondloch, J. E.; Karagiaridi, O.; Bury, W.; Hupp, J. T.; Farha, O. K. Chem. Soc. Rev. 2014, 43, 5896. (b) Cohen, S. M. J. Am. Chem. Soc. 2017, 139, 2855. (c) Li, B.; Zhang, Y.; Ma, D.; Li, L.; Li, G.; Li, G.; Shi, Z.; Feng, S. Chem. Commun. 2012, 48, 6151. (d) Li, F.; Wang, D.; Xing, Q.-J.; Zhou, G.; Liu, S.-S.; Li, Y.; Zheng, L.-L.; Ye, P.; Zou, J.-P. Appl. Catal., B 2019, 243, 621.

[27] Montalbetti, C. A. G. N.; Falque, V. Tetrahedron 2005, 61, 10827.

[28] Canivet, J.; Aguado, S.; Bergeret, G.; Farrusseng, D. Chem. Commun. 2011, 47, 11650.

[29] Bonnefoy, J.; Legrand, A.; Quadrelli, E. A.; Canivet, J.; Farrusseng, D. J. Am. Chem. Soc. 2015, 137, 9409.

[30] Fracaroli, A. M.; Siman, P.; Nagib, D. A.; Suzuki, M.; Furukawa, H.; Toste, F. D.; Yaghi, O. M. J. Am. Chem. Soc. 2016, 138, 8352.

[31] Baek, J.; Rungtaweevoranit, B.; Pei, X.; Park, M.; Fakra, S. C.; Liu, Y.-S.; Matheu, R.; Alshmimri, S. A.; Alshehri, S.; Trickett, C. A.; Somorjai, G. A.; Yaghi, O. M. J. Am. Chem. Soc. 2018, 140, 18208.

[32] Marshall, R. J.; Hobday, C. L.; Murphie, C. F.; Griffin, S. L.; Morrison, C. A.; Moggach, S. A.; Forgan, R. S. J. Mater. Chem. A 2016, 4,6955 .

[33] Gutov, O. V.; Molina, S.; Escudero-Adan, E. C.; Shafir, A. Chemistry 2016, 22, 13582.

[34] Liu, L.; Qiao, Z.; Cui, X.; Pang, C.; Liang, H.; Xie, P.; Luo, X.; Huang, Z.; Zhang, Y.; Zhao, Z. ACS Appl. Mater. Interfaces 2019, 11, 23039.

[35] Liang, K.; Ricco, R.; Doherty, C. M.; Styles, M. J.; Falcaro, P. CrystEngComm 2016, 18, 4264.

[36] Zhang, S.-Y.; Li, D.; Guo, D.; Zhang, H.; Shi, W.; Cheng, P.; Wojtas, L.; Zaworotko, M. J. J. Am. Chem. Soc. 2015, 137, 15406.

[37] Yusran, Y.; Fang, Q.; Qiu, S. Israel J. Chem. 2018, 58, 971.

[38] Zhang, S.; Zheng, Y.; An, H.; Aguila, B.; Yang, C. X.; Dong, Y.; Xie, W.; Cheng, P.; Zhang, Z.; Chen, Y.; Ma, S. Angew. Chem., Int. Ed. 2018, 57, 16754 .

[39] Luo, B.; He, J.; Li, Z.; Lan, F.; Wu, Y. ACS Appl. Mater. Interfaces 
2019, 11, 47218

[40] Ma, Y. F.; Wang, L. J.; Zhou, Y. L.; Zhang, X. X. Nanoscale 2019, $11,5526$.

[41] Okamoto, Y.; Ikai, T. Chem. Soc. Rev. 2008, 37, 2593.

[42] (a) Peluso, P.; Mamane, V.; Cossu, S. J. Chromatogr. A 2014, 1363, 11. (b) Bhattacharjee, S.; Khan, M.; Li, X.; Zhu, Q.-L.; Wu, X.-T. Catalysts 2018, 8, 120.

[43] Zhang, J. H.; Nong, R. Y.; Xie, S. M.; Wang, B. J.; Ai, P.; Yuan, L. M. Electrophoresis 2017, 38, 2513

[44] Kou, W.-T.; Yang, C.-X.; Yan, X.-P. J. Mater. Chem. A 2018, 6, 17861.

[45] Navarro-Sánchez, J.; Argente-García, A. I.; Moliner-Martínez, Y.; Roca-Sanjuán, D.; Antypov, D.; Campíns-Falcó, P.; Rosseinsky, M. J.; Martí-Gastaldo, C. J. Am. Chem. Soc. 2017, 139, 4294.

[46] Huang, K.; Dong, X.; Ren, R.; Jin, W. AIChE J. 2013, 59, 4364

[47] Chan, J. Y.; Zhang, H.; Nolvachai, Y.; Hu, Y.; Zhu, H.; Forsyth, M.;
Gu, Q.; Hoke, D. E.; Zhang, X.; Marriot, P. J.; Wang, H. Angew. Chem., Int. Ed. 2018, 130, 17376.

[48] Lu, Y.; Zhang, H.; Chan, J. Y.; Ou, R.; Zhu, H.; Forsyth, M.; Marijanovic, E. M.; Doherty, C. M.; Marriott, P. J.; Holl Banaszak, M. M.; Wang, H. Angew. Chem., Int. Ed. 2019, 131, 17084.

[49] Mon, M.; Ferrando-Soria, J.; Grancha, T.; Fortea-Perez, F. R.; Gascon, J.; Leyva-Perez, A.; Armentano, D.; Pardo, E. J. Am. Chem. Soc. 2016, 138, 7864 .

[50] Perez-Cejuela, H. M.; Mon, M.; Ferrando-Soria, J.; Pardo, E.; Armentano, D.; Simo-Alfonso, E. F.; Herrero-Martinez, J. M. Mikrochim. Acta 2020, 187, 201.

[51] Feng, X.; Jena, H. S.; Leus, K.; Wang, G.; Ouwehand, J.; Van Der Voort, P. J. Catal. 2018, 365, 36.

[52] Jeong, G. S.; Kathalikkattil, A. C.; Babu, R.; Chung, Y. G.; Won Park, D. Chin. J. Catal. 2018, 39, 63.

(Cheng, B.) 